\title{
The properties of HPMC: PEO extended release hydrophilic matrices and their response to ionic environments
}

Anran $\mathrm{Hu}^{1}$, Chen Chen ${ }^{3}$, Michael D. Mantle ${ }^{3}$, Bettina Wolf ${ }^{2}$, Lynn, F. Gladden ${ }^{3}$, Ali RajabiSiahboomi ${ }^{4}$, Shahrzad Missaghi ${ }^{4}$, Laura Mason ${ }^{1}$, Colin D. Melia ${ }^{1 *}$

${ }^{1}$ Formulation Insights, School of Pharmacy, Nottingham, NG7 2RD, UK, ${ }^{2}$ Food Sciences, School of Biosciences, University of Nottingham, Loughborough LE12 5RD, UK, ${ }^{3}$ Department of Chemical Engineering and Biotechnology, University of Cambridge CB2 3RA, UK, ${ }^{4}$ Colorcon Inc. Global Headquarters, 275 Ruth Road, Harleysville, PA 19438, USA

Key words: Hydrophilic matrix, HPMC, PEO, polymer mixtures, dissolution, MRI, confocal fluorescence microscopy, digital imaging

Abbreviations: Hydroxypropyl methylcellulose (HPMC), polyethylene oxide (PEO), Magnetic resonance imaging $(\mathrm{MRI})$.

\section{Abstract}

Purpose

Investigate the extended release behaviour of compacts containing mixtures of hydrophilic HPMC and PEO in hydrating media of differing ionic strengths.

\section{Methods}

The extended release behaviour of various HPMC:PEO compacts was investigated using dissolution testing, confocal microscopy and magnetic resonance imaging, with respect to polymer ratio and ionic strength of the hydrating media.

Results

Increasing HPMC content gave longer extended release times, but a greater sensitivity to high ionic dissolution environments. Increasing PEO content reduced this sensitivity. The addition of PEO to a predominantly HPMC matrix reduced release rate sensitivity to high ionic environments. Confocal microscopy of early gel layer development showed the two polymers appeared to contribute independently to gel layer structure whilst together forming a coherent and effective diffusion barrier. There was some evidence that poorly swollen HPMC particles added a tortuosity barrier to the gel layer in high ionic strength 
environments, resulting in prolonged extended release. MRI provides unique, non-invasive spatially resolved information from within the HPMC:PEO compacts that furthers our understanding of USP 1 and USP 4 dissolution data.

\section{Conclusions}

Confocal microscopy and MRI data show that combinations of HPMC and PEO have advantageous extended release properties, in comparison with matrices containing a single polymer.

\section{Introduction}

Hydrophilic matrices are a widely-used design of oral extended release dosage form. They can provide prolonged release for drugs with widely different solubilities, and they are simple and easy to formulate. A hydrophilic matrix tablet comprises a drug, standard tableting excipients, and a water swellable polymer which hydrates in gastric media to form a viscous mucilage "gel layer" at the matrix surface. This layer acts as a diffusional and erosional barrier to control further uptake of water into the tablet, and to limit the rate of drug dissolution and release. In simple terms, water soluble drugs are extended by diffusion through the hydrated polymer layer and poorly soluble drugs by matrix erosion [1-5].

A wide range of swelling polymers can successfully be used in hydrophilic matrices. These include many natural, semisynthetic and fully synthetic polymers $[1,6]$. Judicious choice of the polymer and properties such as matrix hydration rate and gel strength, can usually enable desired pharmacokinetic profiles to be achieved [1]. . Hydroxypropyl methylcellulose (HPMC) is the most widely-used. It is available in a variety of viscosity grades, has low toxicity, and there is an extensive literature database [7]. However, HPMC hydration can be influenced by ionic drugs, high concentrations of sugars, and inorganic salts according to their position in the Hofmeister lyotropic series [8]. The effects appear to be related to changes in water structuring and water-polymer binding but multivalent and high strength ionic solutions, can inhibit the formation of an effective surface barrier, and drug release is accelerated $[9,10]$.

This study uses salt concentrations up to $1.0 \mathrm{M} \mathrm{NaCl}$. Whilst this is unlikely to occur in the stomach for a prolonged time, and has been proposed that salt concentrations no higher 
than $0.4 \mathrm{M}$ are tolerated in the fed stomach, this salt is being used as a model ionic challenge to mimic high and low ionic strength in the in vitro drug release studies. The effects of dissolved salts, sugars and other ionic species are cumulative, and mixtures containing multivalent salts, such as sodium citrate can be particularly potent in altering HPMC matrix behaviour. It has been suggested that the effects of soluble species may explain the occasional erratic behaviour of these dosage forms in fed state in vivo studies. Sodium citrate is widely used in cheese. Soft drinks can contain 0.3 to $0.4 \mathrm{M}$ sucrose and preserved foods can contain high amounts of $\mathrm{NaCl}[9,11]$. Variations in $\mathrm{pH}$ are likely to be less problematic, as HPMC and PEO have no ionisable groups and $\mathrm{pH}$ changes within the physiological range do not appear to influence HPMC or PEO matrix gel layer formation. However, it would be reasonable to look at the effects of varying fed stomach $\mathrm{pH}$, in cases where the drug has $\mathrm{pH}$ dependent solubility [12].

Despite their simple formulation, the internal mechanisms that contribute to drug release in hydrophilic matrices are complex. Dynamic processes such as polymer hydration, swelling diffusion and erosion, create water, drug and polymer concentration gradients within the gel layer. As a result, a range of in vitro imaging techniques have been utilised to better understand the internal processes that drive drug release behaviour [1]. Confocal fluorescence microscopy has shown how initial formation of a coherent gel layer is a critical stage in establishing the surface diffusion barrier and the subsequent extended release characteristics [13]. ${ }^{1} \mathrm{H}$ NMR microscopy (magnetic resonance imaging or MRI) has been used to provide spatial and temporal resolved images, over longer timescales and used to monitor the rate of water penetration and mobility [14]. Simple optical images of sectioned tablets can also provide useful information on matrix behaviour when MRI cannot be used, for example in high salt media.

It has been reported that PEO can provide a useful alternative to HPMC in hydrophilic matrix applications, and several studies have compared these two polymers [15-18]. Fasshi et al. have reported that PEO provides faster drug release, relating this to a lower gel strength, greater water uptake, and greater erosion rate of the matrix ${ }_{[19]}$. Whilst many other polymer combinations have been studied, the use of HPMC with PEO as a combined polymer carrier in hydrophilic matrices has received little attention [20-22]. In this study we investigate the properties of matrices containing both HPMC and PEO, with particular respect to their 
extended release behaviour, internal hydration processes and their behaviour in ionic solutions.

\section{Materials and methods}

\subsection{Materials}

Caffeine anhydrous, magnesium stearate, Congo red, concentrated hydrochloric acid (37\%), and sodium chloride were obtained from Sigma-Aldrich (Gillingham, UK). Lactose monohydrate fast flo NF was supplied by Foremost Farms (Baraboo, USA). HPMC was Methoce ${ }^{\circledR}$ K100M CR Premium (BN: Y1090212N01) with a methoxyl content of $22.7 \%$ and hydroxypropoxyl 10.4\%. PEO was POLYOX WSR 303 coagulant PEO NF (BN: 2F1755S5C3). Both polymers were kind gifts from Colorcon Ltd (Dartford, UK). All materials were used as received.

\subsection{Matrix manufacture}

Matrix tablets were prepared by direct compression of powder blends containing by weight $10 \%$ caffeine anhydrous, 30\% polymer, 59.5\% lactose and 0.5\% magnesium stearate. The polymer content was HPMC:PEO combinations at weight ratios of 10:0, 8:2, 6:4, 4:6, 2:8 or $0: 10$. Powder blends were prepared by mixing without magnesium stearate for 20 minutes at 47rpm in a shaker-mixer (Turbula, Willy A. Bachofen Maschinenfabrik AG, Basel, Switzerland), then, and mixing with the magnesium stearate for a further five minutes. Matrices were manufactured by dry compression at a force of $8 \mathrm{kN}$ on a rotary tablet press (Riva Piccola rotary press, Buenos Aeros, Argentina), to form $8 \mathrm{~mm}$ diameter round flat-faced tablets of $250 \pm 3 \mathrm{mg}$.

\subsection{Dissolution testing and calculation of dissolution kinetic parameters}

Matrix drug release kinetics were investigated by dissolution testing in USP apparatus I (baskets) at 100rpm (Prolabo, Dissolutest, France)> experiments were undertaken at $37 \pm 0.5^{\circ} \mathrm{C}$, in $900 \mathrm{ml}$ deionised deaerated water with or without added $\mathrm{NaCl}$ and $\mathrm{pH}$ adjusted to 1.2 with $\mathrm{HCl}$. Drug release was monitored at $273 \mathrm{~nm}$ in $10 \mathrm{~mm}$ quartz flow-through cells, using a closed-loop Agilent automated dissolution system (Agilent 8453, Agilent Technologies, Stockport, UK). The caffeine concentration in each sample was calculated by the Agilent software with reference to a standard calibration curve. 
Dissolution-time curves were fitted to the power law (equation 1) [23], a widely used empirical drug release model for evaluating the kinetics and mechanism of drug release.

$$
\frac{M_{t}}{M_{\infty}}=k t^{n} \quad \text { Equation } 1
$$

$M_{t} / M_{\infty}$ is the fraction of drug released at time $t$, calculated by dividing the mass of drug released at time $t$ by the amount of drug released as $t$ approaches infinity. $k$ is the kinetic or rate constant, and $n$ is the diffusional exponent for drug release. The value of the exponent varies according to the release mechanism and the geometry of the dosage form and it has been used to broadly describe the drug release mechanism (Table 1) [24]. Drug release data between $10 \%-70 \%$ was fitted to this equation ${ }_{[25]}$.

T80 values (the time taken for $80 \%$ drug release) were used a simple measure of extended release and were obtained by interpolation of the release curves [26].

\begin{tabular}{lcccc}
\hline Geometry & \multicolumn{1}{c}{ Case I: } & $\begin{array}{c}\text { Intermediate/ } \\
\text { anomalous } \\
\text { Fickian diffusion } \\
\text { controlled transport }\end{array}$ & $\begin{array}{c}\text { Case II: } \\
\text { Swelling controlled } \\
\text { transport }\end{array}$ & $\begin{array}{c}\text { Super } \\
\text { Case II } \\
\text { transport }\end{array}$ \\
\hline Slab & $n=0.5$ & $0.5<n<1.0$ & $n=1.0$ & $n>1.0$ \\
Cylinder & $n=0.45$ & $0.45<n<0.89$ & $n=0.89$ & $n>0.89$ \\
Sphere & $n=0.43$ & $0.43<n<0.85$ & $n=0.85$ & $n>0.85$
\end{tabular}

Table 1: The effect of changing dosage form geometry on $\boldsymbol{n}$ (diffusional exponent) values for different drug transport mechanisms [24].

\subsection{Magnetic resonance imaging (MRI)}

Experiments were performed on a Bruker AV-400 spectrometer with a vertical $9.4 \mathrm{~T}$ superconducting magnet and micro-imaging facilities. A $25 \mathrm{~mm}$ birdcage radio frequency (r.f.) coil operating at proton $\left({ }^{1} \mathrm{H}\right)$ frequency of $400.23 \mathrm{MHz}$ was used. The $T_{2}$-pRARE $\left(T_{2^{-}}\right.$ preconditioned Rapid Acquisition with Relaxation Enhancement) pulse sequence was used to produce spin-spin relaxation time $\left(T_{2}\right)$ maps, for tablet matrices undergoing drug release in a USP 4 dissolution cell [27-29]. 
$T_{2}$-pRARE images were acquired with, a $64(x$-read $) \times 64(y$-phase $)$ data array with a field of view of $25 \mathrm{~mm} \times 25 \mathrm{~mm}$, yielding an in-plane pixel resolution of $391 \mu \mathrm{m} \times 391 \mu \mathrm{m}$. The slice thickness of these images was $1 \mathrm{~mm}$. The location of the horizontal ( $x y)$ MRI slice was taken through the middle of the tablet which was determined from initial 'pilot' vertical slices $(z x)$ taken at the start of the experiment using the Bruker Biospin Paravision ${ }^{\mathrm{TM}} 4.1$ software interface.

The acquisition bandwidth was $200,000 \mathrm{~Hz}$ and the maximum strength of read gradient, phase gradient and slice gradient were $0.19 \mathrm{~T} / \mathrm{m}, 0.31 \mathrm{~T} / \mathrm{m}$ and $0.14 \mathrm{~T} / \mathrm{m}$ with a RARE factor of 64. Two averages were obtained for each experiment. $T_{1}$ weighting was eliminated by using a recovery time of $10 \mathrm{~s}$ between each $90^{\circ}$ excitation pulse. The temporal resolution of the images was approximately 3 minutes. In accordance with the work of Chen et al., a phase encoding start value of -0.22 was chosen to maximise the quantitative nature of the images [27].

To alleviate artefacts in the spin-spin relaxation time images that arise from fluid motion around the tablet, the images were acquired in the split echo acquisition mode and the signals from the even (effective echo time $=23.34 \mathrm{~ms}, 25.34 \mathrm{~ms}, 29.34 \mathrm{~ms}, 53.34 \mathrm{~ms}, 85.34$ $\mathrm{ms}, 277.34 \mathrm{~ms}, 533.34 \mathrm{~ms}, 1045.34 \mathrm{~ms}$ ) and odd echoes (effective echo time $=23.02,25.02$ ms, 29.02 ms, $53.02 \mathrm{~ms}, 85.02 \mathrm{~ms}, 277.02 \mathrm{~ms}, 533.02 \mathrm{~ms}, 1045.02 \mathrm{~ms}$ ) were added together after Fourier transformation of each group of echoes. $T_{2}$ relaxation maps were produced by analysing the raw data off line using in-house software.

MRI studies of $\mathrm{NaCl}$ solutions could not be undertaken in the spectrometer because the 25 $\mathrm{mm}$ birdcage r.f. coil used in this study cannot be tuned to working ${ }^{1} \mathrm{H}$ frequency in the presence of excess dielectric influences.

A schematic of the combined MRI/UV-vis. experimental set-up is shown in Figure 1. A standard USP-IV flow-through dissolution cell (internal diameter $22.6 \mathrm{~mm}$ ) (Sotax, Switzerland) was mounted within the magnetic resonance spectrometer, and used for the tablet dissolution study following the method of Zhang et al. [28]. The medium was $500 \mathrm{~mL}$ water adjusted to $\mathrm{pH} 1.2$ as described above, with UV detection of drug at $273 \mathrm{~nm}$. A flow rate of $8 \mathrm{ml} / \mathrm{min}$ (peristaltic pump 205S, Watson Marlow, USA) and a temperature of $37^{\circ} \mathrm{C}$ 
was maintained. The tablet was placed vertically in the USP-IV dissolution cell, with its crosssection selected as the imaging plane.

The drug release profile was measured on-line with the UV-Vis spectrometer (Spectronic Unicam, UK) with a quartz cell (path length $=5 \mathrm{~mm}$ ). Absorption was measured at a wavelength of $273 \mathrm{~nm}$ at a resolution of $1 \mathrm{~nm}$ and the concentration of caffeine in solution was calculated using a prepared calibration curve with caffeine concentrations between $0.005 \mathrm{mmol} / \mathrm{L}$ and $0.15 \mathrm{mmol} / \mathrm{L}$. The measurements were carried out using VisionLite software (Thermo Scientific).

\subsection{Confocal laser scanning microscopy:}

The formation and development of the initial gel layer at the radial periphery of the tablet matrix were studied by the method of Bajwa et al. [13]. A Bio-Rad MRC-600 confocal microscope (Hemel Hempstead, UK) equipped with a $15 \mathrm{~mW}$ Krypton Argon laser, a Nikon Optiphot upright microscope, and 4x/0.13NA plan air objectives was used. The experimental geometry (figure 2) shows how the tablet was fixed between two Perspex discs held by three acrylic screws in a Fixed Observational Geometry (FOG) sample cell, as designed by Bettini et al [30]. The FOG cell was placed within a temperature controlled $\left(37 \pm 1^{\circ} \mathrm{C}\right)$ vessel and immersed in a hydration fluid containing $0.008 \% \mathrm{w} / \mathrm{v}$ Congo red, a cellulose-active fluorophore which highlights hydrated HPMC within the gel layer (24). Instrument settings were: excitation laser line 488nm, BHS filter block (Ex 488/Em >510nm), confocal aperture 5.0, black level 5.1 (on a scale of 0-255) and a gain level (image brightness) setting of 9.1. Images were obtained at intervals up to 15 minutes.

\subsection{Digital optical imaging}

The matrix tablet was fixed using double sided tape to a $22 \times 22 \mathrm{~mm}$ glass cover slip, and placed into $900 \mathrm{ml}$ media containing $\mathrm{NaCl}(0 \mathrm{M}, 0.4 \mathrm{M}$ and $1.0 \mathrm{M})$ with a paddle speed of $50 \mathrm{rpm}$ to simulate the tablet dissolution process. Tablets were taken from the media, and placed under the digital camera after $0.5,1,2,4,6$ and 8 hours hydration. Images of the hydrated tablets, before and after axial sectioning, were obtained against a black background, under standardised lighting conditions, using a Cool Snap Pro cf camera (Media Cybernetics $^{\mathrm{TM}}$ ) fitted with a Nikon AF Micro Nikkor $600 \mathrm{~mm}$ macro lens. 


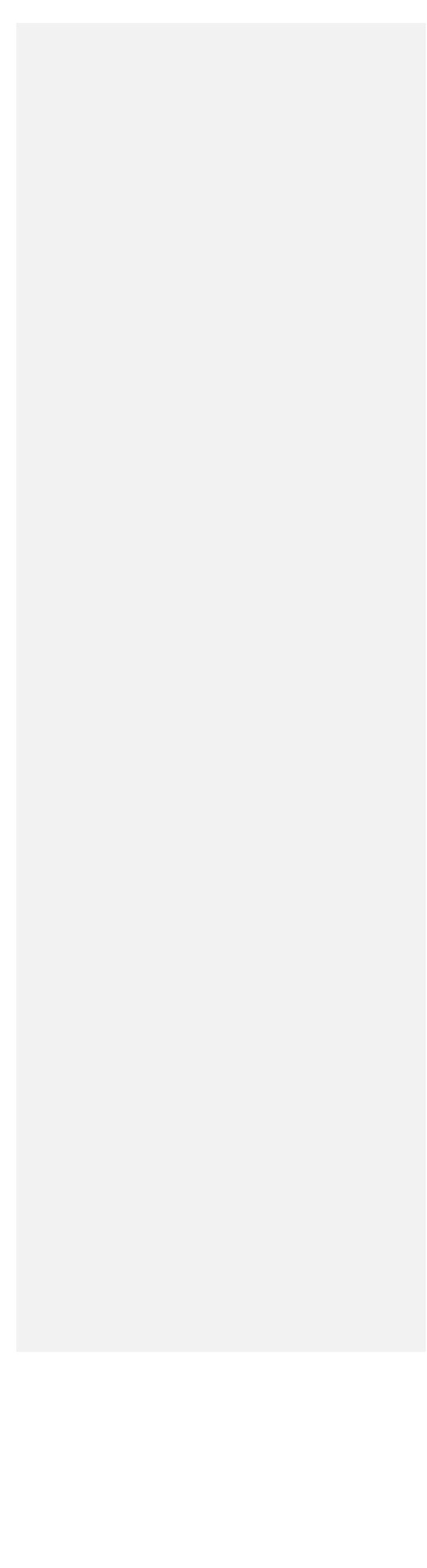




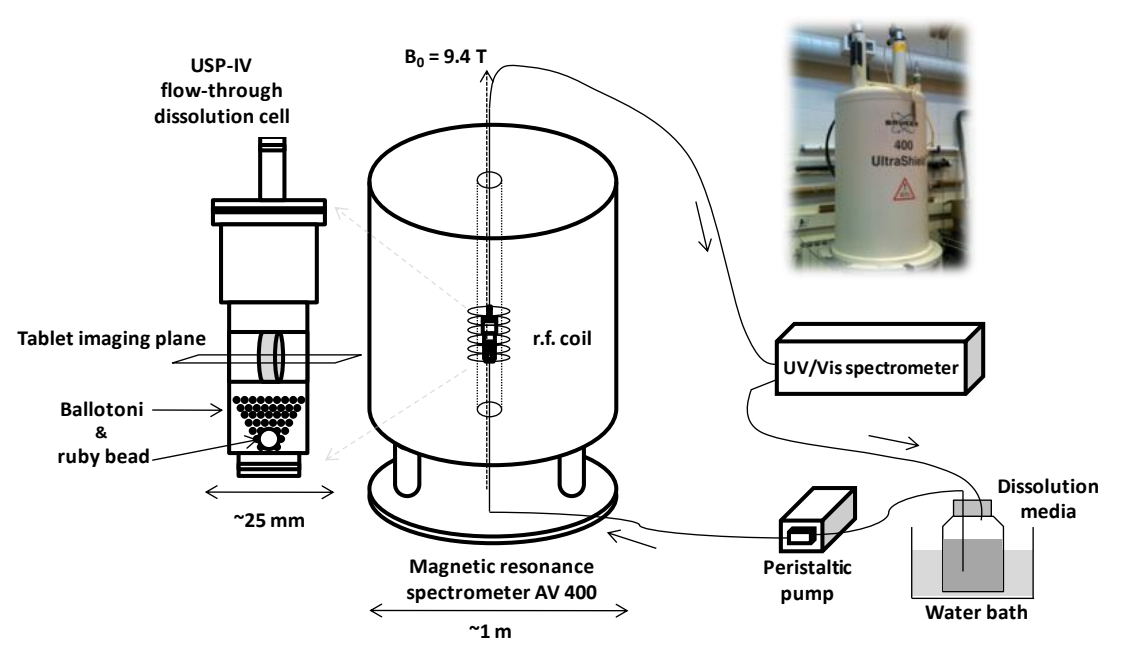

Figure 1: Schematic diagram showing the experimental geometry for MRI imaging. The loaded USP- 4 flow cell was inserted into the spectrometer inside the rf coil. The hydration medium was maintained at $37 \pm 1^{\circ} \mathrm{C}$.

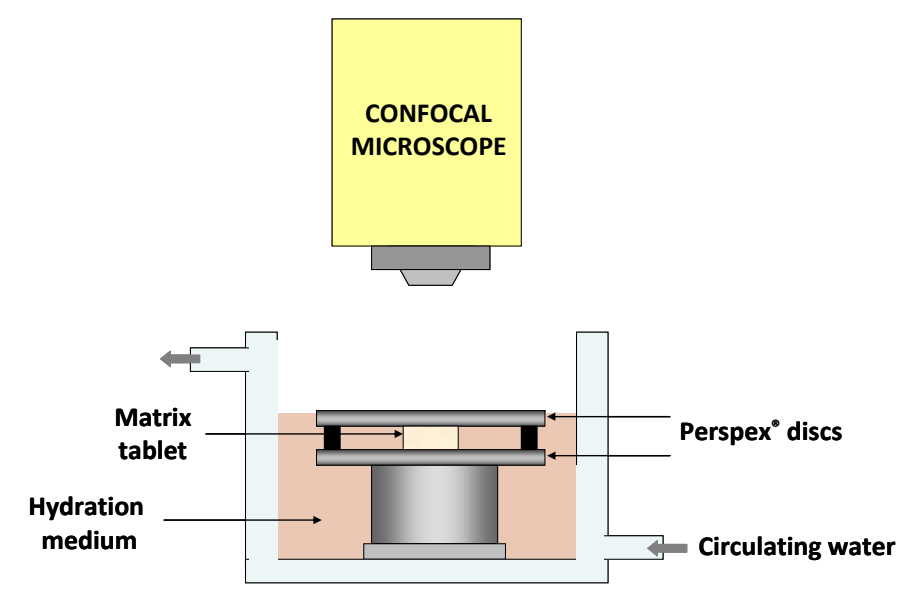

Figure 2: Schematic diagram showing the Fixed Observational Geometry cell used for confocal imaging.

The hydration medium was maintained at $37 \pm 1^{\circ} \mathrm{C}$. 


\section{Results and Discussion}

\subsection{In-vitro drug release}

\subsubsection{Dissolution curves}

Figure 3 shows matrix dissolution test results for different ratios of HPMC and PEO. Total polymer content was maintained at $30 \% \mathrm{w} / \mathrm{w}$ throughout. The $\mathrm{NaCl}$ concentration in the dissolution medium was varied in order to show the effect of ionic strength on drug release. Although the salt concentrations used here are considered abnormally high for physiological and dietary relevance, $\mathrm{NaCl}$ is being used here as a model ionic challenge, to elicit Hofmeister effects that might occur at lower concentrations of multivalent ions and drugs $[8$, 31-34].

All dissolution curves appeared to be similar in shape, except for matrices containing predominately HPMC. Matrices containing HPMC or an 8:2 ratio of HPMC:PEO showed extended release failure at high salt concentrations, and those containing 6:4 HPMC:PEO showed changes in their extended release profiles at the highest salt concentrations.

To examine comparative trends across the range of polymer ratios, values of T80 and power law kinetic parameters ( $k$ and $n$ ) were calculated as previously described.

\subsubsection{T80 values}

T80 values are shown in Table 2 to provide a simple measure of extended release. They show how, in the absence of added $\mathrm{NaCl}, \mathrm{HPMC}$ matrices exhibited slower drug release than PEO matrices. In the presence of up to $0.4 \mathrm{M} \mathrm{NaCl}$, matrices containing HPMC and 8:2 HPMC:PEO, show increased T80 values, but at $0.8 \mathrm{M}$ these matrices exhibit a marked acceleration of drug release. This effect has been previously reported in other HPMC matrix formulations [9] and has been attributed to the failure of an effective gel layer diffusion barrier, during the early stages of matrix hydration, as a result of inhibition of polymer swelling through Hofmeister effects $[35,36]$.

In contrast, PEO matrices retained their capability to extend drug release at all salt concentrations. PEO hydration is reported to be sensitive to salts [35-38], but in these investigations the threshold concentration was clearly much higher for PEO than for HPMC. Dose dumping was not observed in PEO matrices and increasing $\mathrm{NaCl}$ concentration increased T80 from 237 minutes at $0 \mathrm{M} \mathrm{NaCl}$, to 292 minutes in $1.0 \mathrm{M} \mathrm{NaCl}$. Matrices 
containing HPMC:PEO polymer combinations showed decreased extended release times as the amount of PEO was increased, but by using a ratio of at least 6:4 HPMC: PEO the saltinduced rapid release of HPMC matrices was also abolished. Overall, the effect of mixing these two polymers appeared to be additive rather than synergistic.

\subsubsection{Power Law analysis}

Table 3 shows the calculated power law parameters. Correlation coefficient values $\left(r^{2}\right)$ close to 0.999 show that the power law was a reasonable fit to the dissolution curves over the range $10-70 \%$. In media containing up to $0.4 \mathrm{M} \mathrm{NaCl}$, matrices exhibit diffusion exponent values $(n)$ between 0.68 to 0.81 . Values between 0.45 to 0.89 indicate an 'anomalous nonFickian transport' drug release mechanism in which drug release involves a coupling of drug diffusion with polymer relaxation [39]. Matrices in higher $\mathrm{NaCl}$ media, also had $\mathrm{n}$ values in this range. In matrices that disintegrated (8:2 HPMC: PEO or HPMC in $0.8 \mathrm{M} \mathrm{NaCl}$ or above) $n$ values were $>0.89$ a mechanism known as case II transport that depends on polymer relaxation rate.

\subsubsection{A comparison of mixed and single polymer matrices}

The results in Table 2 suggest that matrices containing a 4:6 HPMC:PEO ratio provide a good compromise between maximising extended release, and minimising drug release variability across the salt concentration range. The 4:6 HPMC:PEO matrix was therefore chosen for further investigations to better understand drug release behaviour. Figures 4 show a comparison of this mixed polymer matrix with those containing the single polymers, and it allows us to examine the dissolution curves in greater detail. In $0 \mathrm{M}$ and $0.4 \mathrm{M} \mathrm{NaCl}$ (figure 4 $A$ and $B$ ), all three formulations provide similar drug release kinetics over the first 120 minutes, only then do the profiles diverge. The greater prolongation of drug release in HPMC matrices was therefore an effect only exerted in the later stages of dissolution. In 1.0M NaCl (figure $4 \mathrm{C}$ ) the HPMC matrix exhibited accelerated drug release, whilst the PEO and 4:6 mixed polymer matrices maintained extended release profiles. Strangely, the mixed polymer matrix provided greater extended release than PEO alone, suggesting that the PEO was not only protecting the HPMC from salt sensitivity, but that the HPMC was also able to contribute to extended release. 


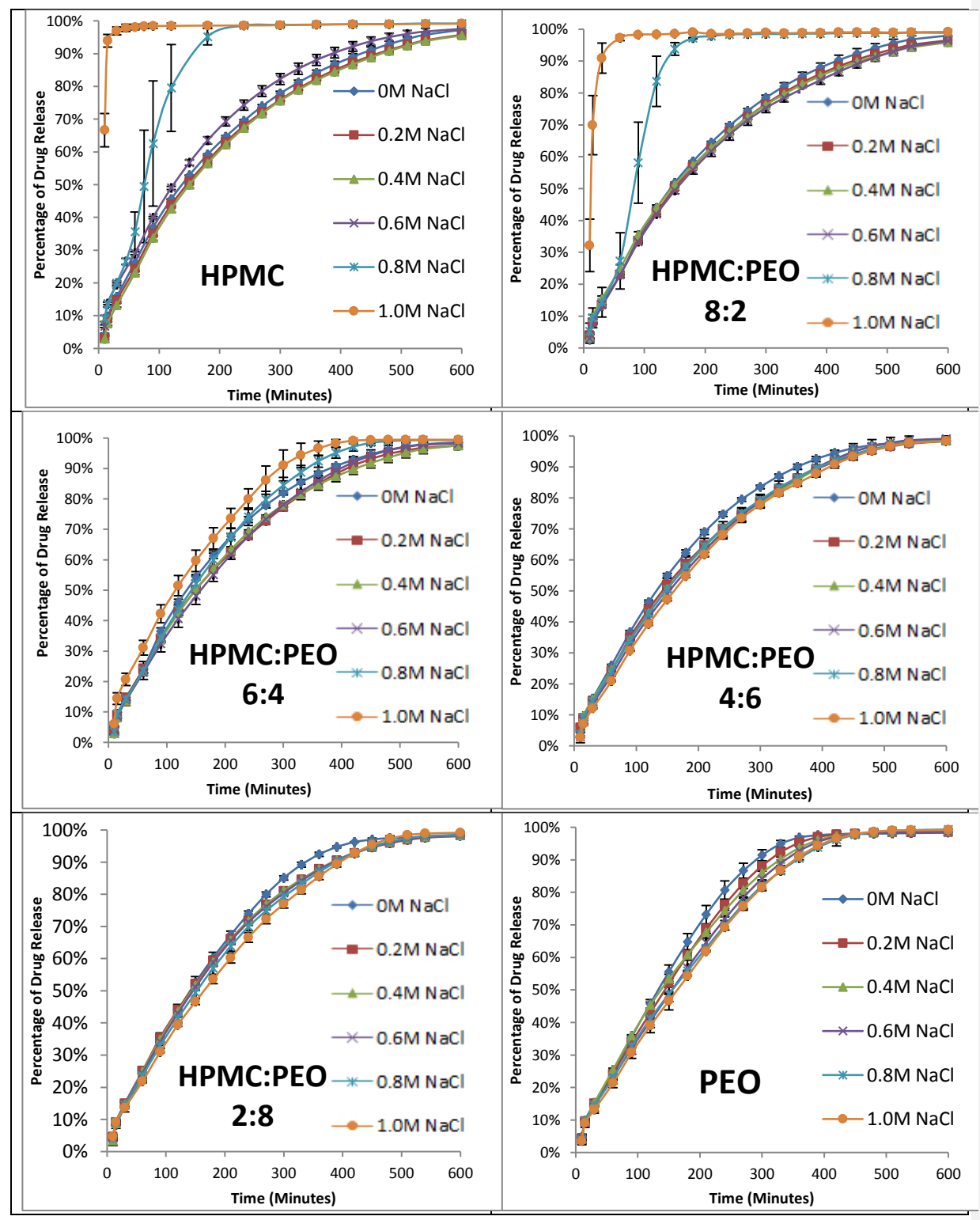

Figure 3: Release of caffeine from hydrophilic matrix tablets as a function of polymer composition and sodium chloride concentration in the dissolution medium $(\mathbf{p H}=\mathbf{1 . 2})$. All matrices contain $30 \% \mathrm{w} / \mathrm{w}$ polymer. USP apparatus $1,100 \mathrm{rpm}, 900 \mathrm{ml}, 37 \pm 0.5^{\circ} \mathrm{C}$. Mean $(\mathrm{n}=3) \pm 1$ SD. 


\begin{tabular}{|c|c|c|c|c|c|c|}
\hline & \multicolumn{5}{|c|}{ NaCl concentration in dissolution medium } \\
& 0 & $0.2 \mathrm{M}$ & $0.4 \mathrm{M}$ & $0.6 \mathrm{M}$ & $0.8 \mathrm{M}$ & \multicolumn{1}{c|}{$1.0 \mathrm{M}$} \\
\hline HPMC & $\mathbf{3 2 0}$ & $\mathbf{3 3 6}$ & 341 & 282 & 120 & 12 \\
\hline HPMC:PEO 8:2 & $\mathbf{3 1 2}$ & $\mathbf{3 3 0}$ & $\mathbf{3 3 0}$ & $\mathbf{3 4 6}$ & 116 & 22 \\
\hline HPMC:PEO 6:4 & 285 & $\mathbf{3 2 0}$ & $\mathbf{3 1 7}$ & $\mathbf{3 1 4}$ & 270 & 240 \\
\hline HPMC:PEO 4:6 & 270 & $\mathbf{3 0 8}$ & $\mathbf{3 1 1}$ & $\mathbf{3 0 8}$ & $\mathbf{3 0 5}$ & $\mathbf{3 1 7}$ \\
\hline HPMC:PEO 2:8 & 270 & 293 & 292 & 297 & $\mathbf{3 0 6}$ & $\mathbf{3 2 1}$ \\
\hline PEO & 237 & 257 & 267 & 278 & 289 & 292 \\
\hline
\end{tabular}

Table 2: T80 values (minutes) with respect to polymer composition and sodium chloride concentration. T80 (the time for $80 \%$ drug release) was obtained from interpolation of the drug release curves. Values in bold italics highlight T80 extended release greater than 6 hours.

\begin{tabular}{|c|c|cccccc|}
\hline \multicolumn{2}{|c}{} & \multicolumn{5}{|c|}{ NaCl concentration in the dissolution medium } \\
\hline HPMC & $k$ & 0 & $0.2 \mathrm{M}$ & $0.4 \mathrm{M}$ & $0.6 \mathrm{M}$ & $0.8 \mathrm{M}$ & $1 \mathrm{M}$ \\
& $n$ & 0.689 & 0.693 & 0.727 & 0.655 & 1.067 & $\mathrm{~N} \backslash \mathrm{A}$ \\
& $r^{2}$ & 0.9969 & 0.9961 & 0.9953 & 0.9980 & 0.9743 & $\mathrm{~N} \backslash \mathrm{A}$ \\
\hline HPMC:PEO & $k$ & 1.071 & 1.245 & 1.649 & 1.261 & 0.122 & $\mathrm{~N} \backslash \mathrm{A}$ \\
8:2 & $n$ & 0.767 & 0.731 & 0.680 & 0.726 & 1.363 & $\mathrm{~N} \backslash \mathrm{A}$ \\
& $r^{2}$ & 0.9955 & 0.9961 & 0.9969 & 0.9967 & 0.9633 & $\mathrm{~N} \backslash \mathrm{A}$ \\
\hline HPMC:PEO & $k$ & 1.065 & 1.305 & 1.085 & 1.058 & 0.954 & 2.200 \\
6:4 & $n$ & 0.780 & 0.724 & 0.761 & 0.760 & 0.797 & 0.658 \\
& $r^{2}$ & 0.9973 & 0.9983 & 0.9983 & 0.9996 & 0.9990 & 0.9984 \\
\hline HPMC:PEO & $k$ & 1.149 & 1.233 & 1.254 & 0.986 & 1.148 & 0.741 \\
4:6 & $n$ & 0.768 & 0.741 & 0.736 & 0.777 & 0.753 & 0.807 \\
& $r^{2}$ & 0.9990 & 0.9974 & 0.9993 & 0.9991 & 0.9992 & 0.9993 \\
\hline HPMC:PEO & $k$ & 0.936 & 1.236 & 1.172 & 1.127 & 1.055 & 1.005 \\
$2: 8$ & $n$ & 0.800 & 0.744 & 0.753 & 0.759 & 0.767 & 0.765 \\
& $r^{2}$ & 0.9989 & 0.9992 & 0.9994 & 0.9994 & 0.9995 & 0.9992 \\
\hline PEO & $k$ & 0.854 & 0.915 & 1.174 & 0.884 & 1.045 & 0.796 \\
& $n$ & 0.802 & 0.807 & 0.760 & 0.801 & 0.766 & 0.813 \\
& $r^{2}$ & 0.9991 & 0.9986 & 0.9993 & 0.9990 & 0.9990 & 0.9987 \\
\hline
\end{tabular}

Table 3: Kinetic parameters obtained from fitting the Power law equation to the drug release curves. Rate constants $(k)$, exponent value $(n)$, goodness of fit $\left(r^{2}\right)$. Data from figure 3 . 

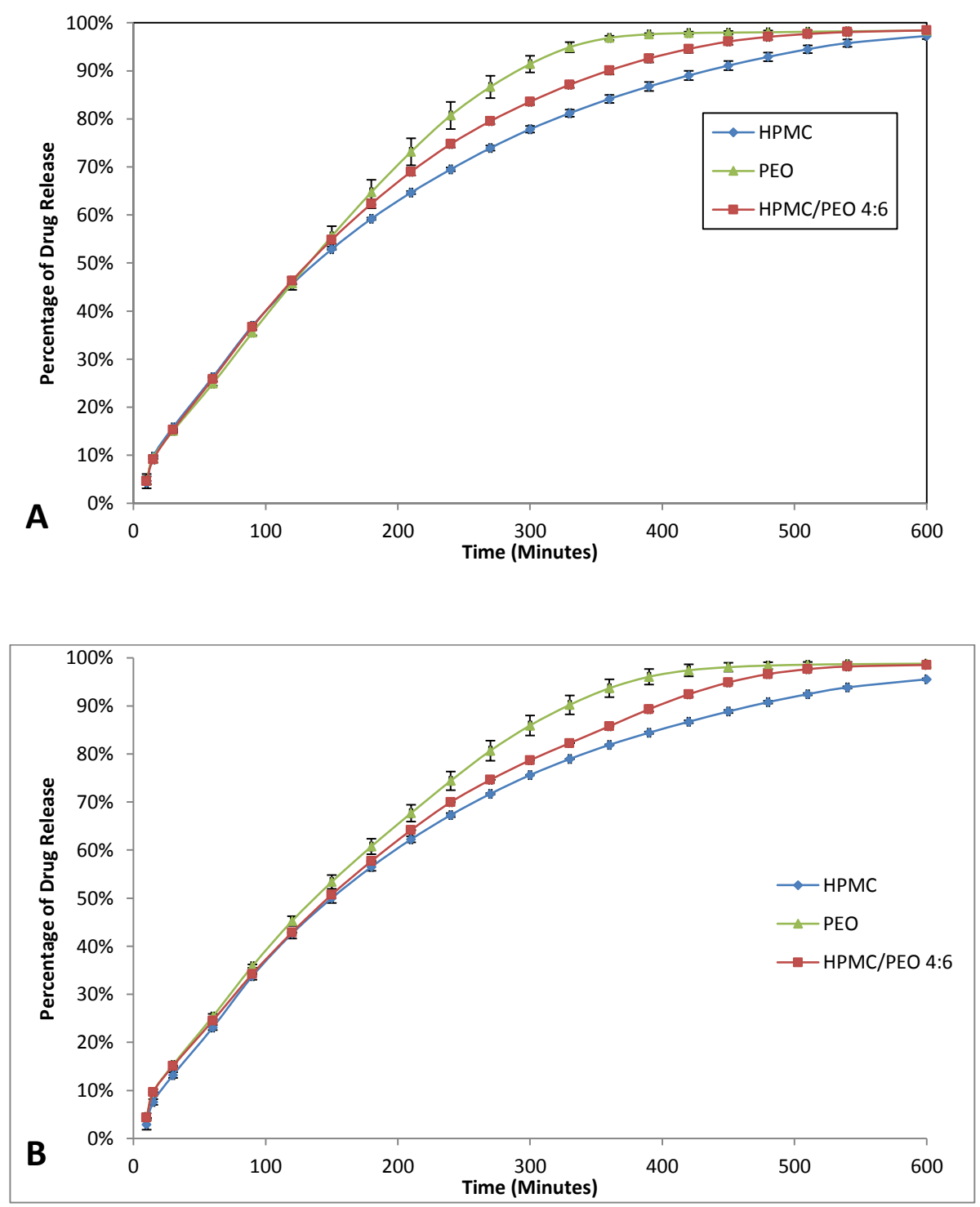


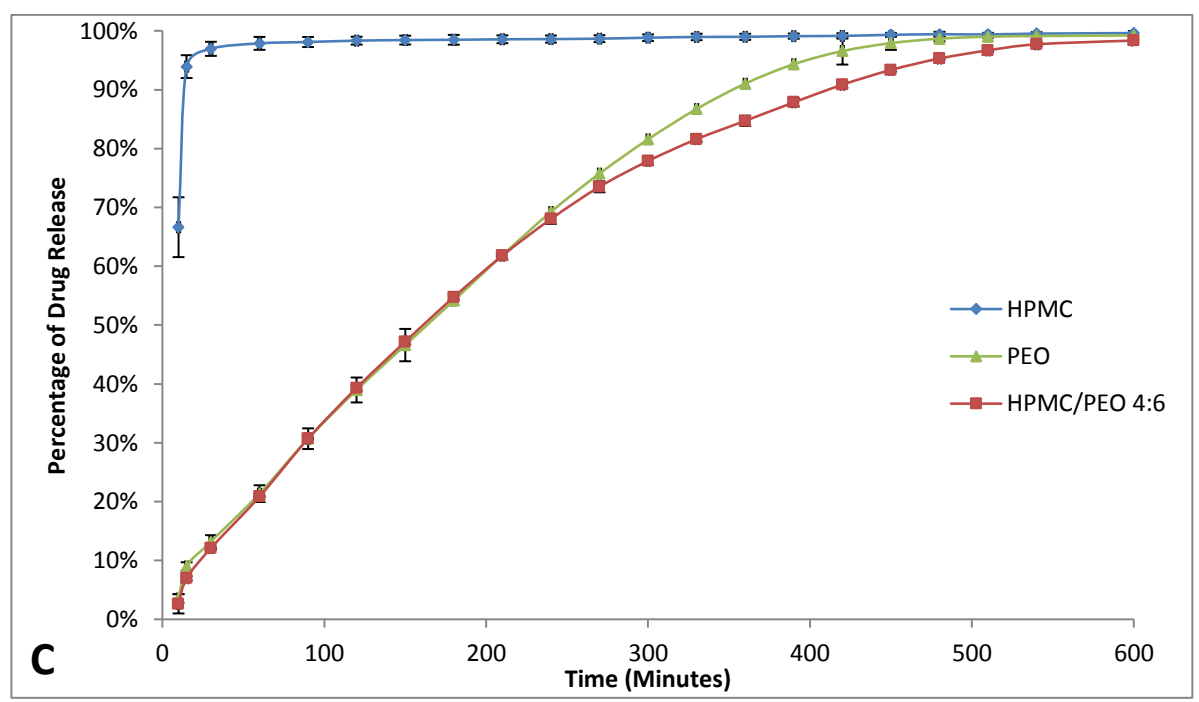

Figure 4: Release of caffeine from single and 4:6 HPMC:PEO mixed polymer matrices in $0 \mathrm{M}(\mathrm{A}), 0.4 \mathrm{M}$ (B) and 1.0M (C) NaCl dissolution medium ( $\mathrm{pH}=1.2)$. All matrices contain 30\%w/w polymer. USP apparatus $1,100 \mathrm{rpm}$, $900 \mathrm{ml}, 37 \pm 0.5^{\circ} \mathrm{C}$. Mean ( $\left.\mathrm{n}=3\right) \pm 1 \mathrm{SD}$. 


\subsection{Magnetic resonance imaging studies of internal hydration behaviour}

Figure 5 shows axial, $1 \mathrm{~mm}$ thick cross-sectional images obtained from matrices swelling in the USP 4 cell in the MRI spectrometer. These spatially resolved images are colour-coded for $T_{2}$ relaxation times and provide semi-quantitative visual maps for comparisons of polymer swelling behaviour, gel layer formation and the progress of internal hydration [27, 28]. The areas coded black relate to the driest regions of the matrix, and can be used to assess the relative shrinkage of the 'dry' core.

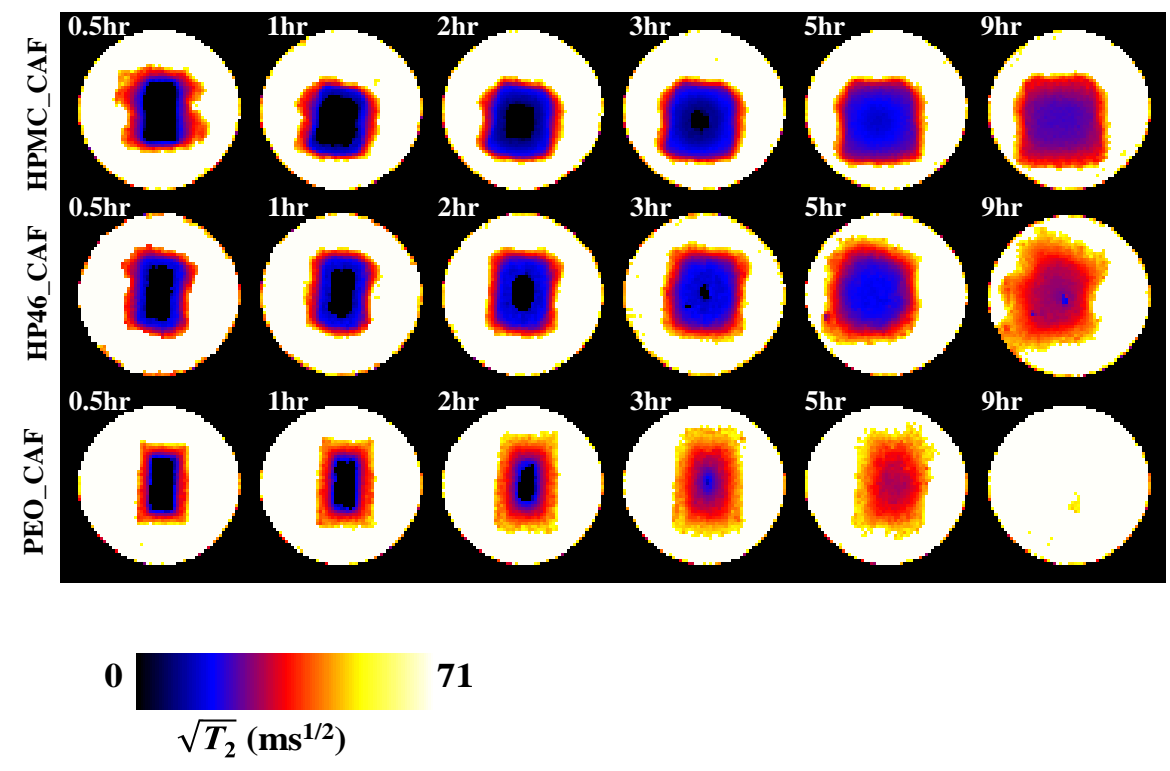

Figure 5: MRI cross-sectional images of matrix hydration over 9 hours. $T_{2}$ relaxation time maps of HPMC, HPMC:PEO 4:6 and PEO hydrophilic matrices in USP 4 dissolution cell in $500 \mathrm{ml}$ water adjusted to $\mathrm{pH} 1.2$, $8 \mathrm{ml} / \mathrm{min}$., $\mathrm{T}=\mathrm{T} 37 \pm 1^{\circ} \mathrm{C}$. Each image is $25 \mathrm{~mm}$ in diameter. Colour coding for $T_{2}$ is shown in the colour bar.

The images show substantially different hydration characteristics in HPMC and PEO matrices. The HPMC matrix: (i) adopts a dumb-bell shape in the early stages; (ii) shows anisotropic swelling in the early stages; (iii) exhibits relatively slow water ingress and (iv) retains a matrix shaped hydrated mass of gelled polymer at 9 hours. Features (i) and (ii) are typical behaviour for HPMC-based matrices [27] with the dumb-bell shape and axial swelling arising from uniaxial relaxation of elastic energy stored during compression [40]. 
In contrast, the PEO matrix shows: (i) less dimensional changes than HPMC during the first hour of hydration; (ii) Isotropic swelling behaviour; (iii) a more rapid water ingress into the matrix and (iv) the complete dissolution of the matrix at 9 hrs. Features (i) and (ii) reflect the low levels of elastic energy stored during PEO deformation, whereas (iii) and (iv) are consistent with the higher water affinity of PEO [41]. This arises because PEO has no hydrophobic substituents whereas Methocel K100M HPMC (USP type 2208) has 22\% methoxyl substitution.

Images of the 4:6 HPMC:PEO matrix show hydration behaviour intermediate between HPMC and PEO: (i) a reduced axial swelling in comparison with HPMC; (ii) a reduced water ingress with respect to PEO and (iii) a matrix that retains some integrity at $9 \mathrm{hrs}$. This evidence suggests that substituting HPMC in the PEO formulation slows water penetration, and at the end of the test has a highly hydrated matrix that retains some shape. Both features may give the matrix a greater longevity, consistent with the results of dissolution testing (Figure 4A). Calculation of dissolution parameters from the later part of the dissolution curve show that HPMC containing matrices exhibit $n$ values consistent with diffusion dominated release, whereas PEO matrices exhibit $n$ values that suggest a greater contribution from surface erosion (Table 4). This would account for the disappearance of the PEO gel mass at the end of the dissolution test.

\subsubsection{Correlating in-line USP-4 drug release curves with MRI data.}

Figure 6 shows the results from the on-line UV-vis analysis of the effluent stream from the MRI/USP-4 dissolution cell used to obtain the images depicted in figure 5 . 


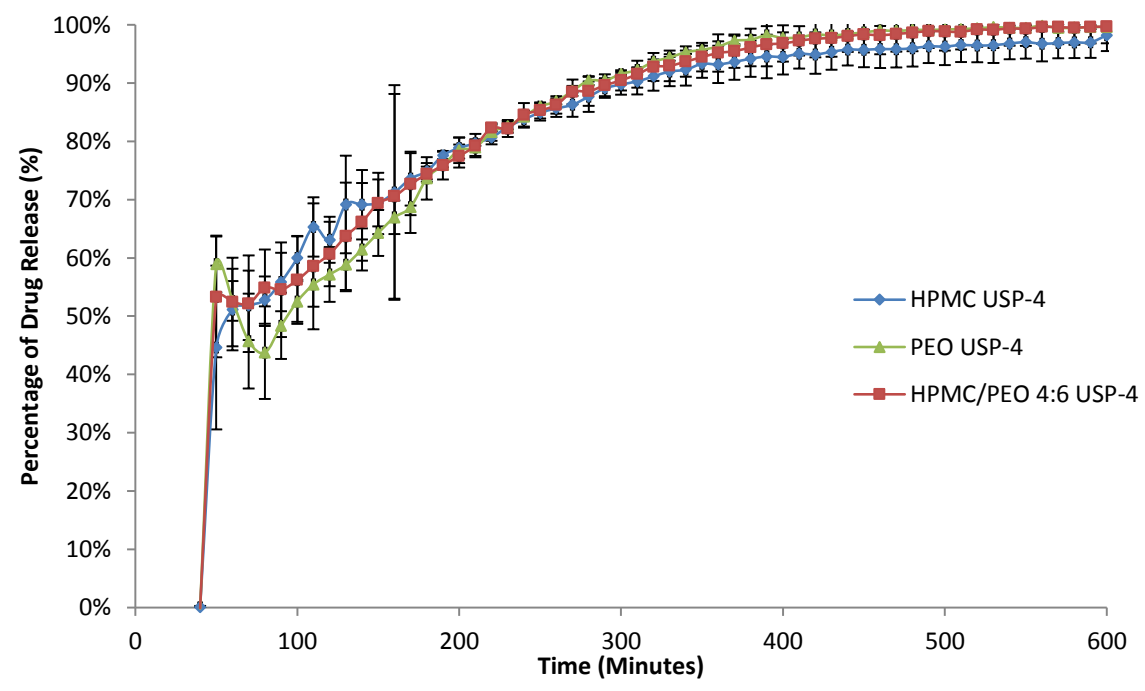

Figure 6: Release of caffeine from the on-line MRI/UV-vis apparatus for individual tablets of HPMC, 4:6 HPMC:PEO and PEO in pH 1.2 water based dissolution medium. All matrices contain $30 \% \mathrm{w} / \mathrm{w}$ polymer. USP apparatus $4,8 \mathrm{ml} / \mathrm{min}, 500 \mathrm{ml}, 37 \pm 1^{\circ} \mathrm{C}$. Mean ( $\left.=3\right) \pm 1 S D$.

The images for HPMC and 4:6 HPMC: PEO at $30<t<180$ minutes (figure 5) demonstrate a difference in the rate of disappearance of the dry core (black coloured area within the tablet) with a rank order PEO > 4:6 HPMC:PEO > HPMC. However, there is little visible difference in the overall size of the gel layer for HPMC and 4:6 HPMC:PEO samples (red coloured areas up to $t=120$ minutes). The equivalent USP 4 data (figure 6 ) are too noisy at this stage to give a statistically meaningful interpretation of the observed drug release rates with respect to the MRI images shown in figure 5. Figure 5 shows a transition in the appearance of the gel layer occurs at $t=180$ minutes. At $t=300$ minutes there is a distinct change in the physical distribution of $T_{2}$ values for HPMC and 4:6 HPMC:PEO tablets, with 4:6 HPMC:PEO showing a greater proportion of higher $T_{2}$ values for the water (red/yellow colouring) and is indicative of system with a greater water/polymer mobility which inturn correlates with a greater amount of drug release. This observation is further supported by the HPMC and 4:6 HPMC:PEO MRI images at $t=420$ minutes and 540 minutes. For $t>300$ mins figure 5 also shows an increase in tablet surface area when comparing 4:6 HPMC:PEO with HPMC. The changes in gel layer morphology (assed using the $T_{2}$ values) and physical surface area for HPMC and 4:6 HPMC:PEO for $t>300$ minutes correlates well with the on- 
line USP 4 dissolution data shown in figure 6 which shows an enhanced drug release for the 4:6 HPMC:PEO system relative to HPMC alone for $300<t<540$ mins. For PEO tablets, there is a dramatic change in the $T_{2}$ values at $\mathrm{t}=300$ minutes indicating a system that has a higher polymer mobility compared to the HPMC and 4:6 HPMC:PEO samples. The onset of an additional erosion mechanism of the PEO matrix is also clearly evident for $t>300$ minutes and both the higher $T_{2}$ values and erosion result in a higher rate of drug release relative to the HPMC/4:6 HPMC:PEO tablets; this behaviour again correlates well with the on-line USP 4 shown in figure 6. Despite a small time offset, the coupled MRI/USP 4 observations discussed above are qualitatively consistent with the independent USP 1 drug release data shown in figure $4 \mathrm{~A}$, and thus highlight the power of MRI in translating the physical reasons for the differences in standard USP type drug release curves.

\begin{tabular}{|l|c|c|}
\hline & \multicolumn{1}{|c|}{$\begin{array}{c}\text { Time between } \\
\text { T60-T95\% } \\
\text { (minutes) }\end{array}$} & $\begin{array}{c}\text { Power law exponent } \\
n\end{array}$ \\
\hline HPMC matrix & 360 & 0.41 \\
\hline 4:6 HPMC:PEO matrix & 270 & 0.45 \\
\hline PEO matrix & 180 & 0.63 \\
\hline
\end{tabular}

Table 4: Dissolution parameters calculated over the range $60-95 \%$ drug release. Parameters calculated from dissolution data in figure 4 . 


\subsection{Confocal fluorescence microscopy studies of early gel layer formation and growth}

Confocal microscopy imaging was used to compare early gel layer initiation and development. Studies were undertaken in media containing $0 \mathrm{M}, 0.4 \mathrm{M}$ and $1.0 \mathrm{M} \mathrm{NaCl}$. 0.4 $\mathrm{M} \mathrm{NaCl}$ represents the longest extended release obtained for HPMC matrices, and 1.0M was an extreme concentration that resulted in burst release.

\subsubsection{Early gel development in media without sodium chloride}

Figure 7 shows typical gel formation in media without $\mathrm{NaCl}$. The extent of gel growth can be seen from the dashed line, which represents the unhydrated tablet perimeter. HPMC matrices exhibit an uninterrupted high intensity fluorescent boundary at the gel layer periphery, where highly swollen and disentangling polymer allows easy access of binding sites to the fluorophore ${ }^{[13,42]}$. A pattern of fluorescence is also identifiable within the inner regions of the hydrated gel layer, and is more heterogeneous because the fluorophore has penetrated initially through tablet capillaries, which then become blocked [13]. The fluorophore also highlights the emerging gel layer in the PEO matrix, but the fluorescent intensity was lower, and more uniformly distributed. This suggested the rapid diffusion through, and a lack of specific binding, of Congo red in the gel layer, However fluorescence intensity was clearly discernible above background.

This contrast in fluorescent intensity, between the PEO and HPMC matrices, was useful in distinguishing these two polymers in the gel layer of the 4:6 HPMC:PEO matrix. This showed regions of high intensity, most likely swelling HPMC particles, separated and interspersed by swollen regions of low fluorescence, which most likely represent the PEO. There was no evidence of significant polymer mixing, even in the most hydrated outermost part of the gel layer.

In addition, the outer part of the gel layer shows HPMC particles are highly swollen, whilst the inner regions show only outlines of HPMC particles which are poorly swollen because they have only a marginally higher fluorescence. This may have arisen from poor fluorophore penetration but we feel this is unlikely because of the freedom with which Congo red can diffuse through swollen PEO. This result raises the possibility that PEO swells preferentially in the inner regions of the gel, and dominates the water availability, whilst at 
the outer surface of the gel layer, HPMC particles can swell freely. The suppression of HPMC swelling by a different grade of PEO has been reported previously [21].

All matrices showed the rapid formation of a gel diffusion barrier, with little evidence of significant liquid penetration of the core. The effectiveness of this diffusion barrier in the mixed HPMC:PEO matrix, suggests that both polymers contribute to a continuous and coherent gel layer.

PEO matrices exhibited a greater overall swelling than other matrices, supporting the idea that the gel layer may become more rapidly established as a result of the greater water affinity of this polymer

\subsubsection{Early gel layer development in sodium chloride solutions}

Figure 8 shows images of gel layer formation in $0.4 \mathrm{M} \mathrm{NaCl}$. They show a similar pattern of swelling and early gel layer development to matrices hydrating without $\mathrm{NaCl}$.

Figure 9 shows images of matrices hydrated in $1 \mathrm{M} \mathrm{NaCl}$. This extreme concentration was chosen to disrupt HPMC particle coalescence and this is clearly seen in the extensive swollen mass of individual particles that have replaced the gel layer. This pattern signifies failure to establish a diffusion barrier that can block water penetration [10] and in the dissolution tests, these HPMC matrices released drug rapidly (Table 2). In contrast, the PEO matrix shows gel layer formation progressing normally, apparently unaffected by the high salt concentration and in the dissolution tests, these matrices retained their extended release properties (Table 2).

In the mixed HPMC: PEO matrices, HPMC particles within the emerging gel layer exhibit low fluorophore uptake. They appear to be surface stained but not highly swollen. This suggests that these HPMC regions are poorly hydrated as a result of the high salt concentration. However, gel layer diffusion barrier properties remain intact and dissolution tests in $1.0 \mathrm{M}$ $\mathrm{NaCl}$ show this matrix has better extended release characteristics than a matrix based on PEO alone. This suggests that the poorly hydrated HPMC might add a tortuosity factor to the diffusional release of drug through the gel layer, acting in a similar way to insoluble inclusions such as microcrystalline cellulose [43]. This might account for the slower release profiles of mixed matrices seen in the dissolution tests (figure $4 \mathrm{C}$ ). 


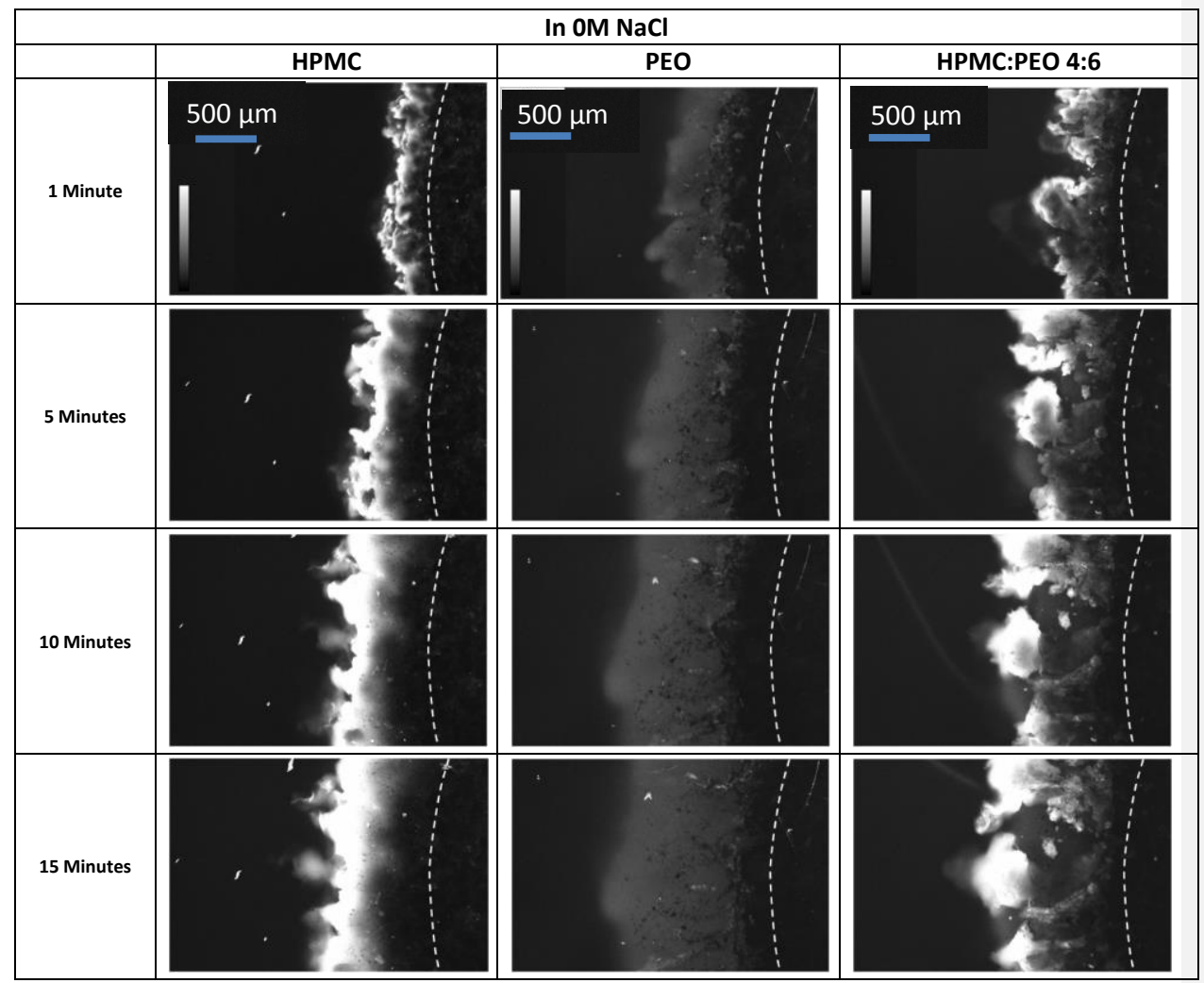

Figure 7: Early gel layer growth in the absence of $\mathrm{NaCl}$. Confocal fluorescence imaging at $\mathrm{Ex} 488 / \mathrm{Em} 510 \mathrm{~nm}$. Experiments conducted at $37 \pm 1{ }^{\circ} \mathrm{C}$ using $\mathrm{NaCl}$ media containing $0.008 \% \mathrm{w} / \mathrm{v}$ Congo red as a visualisation aid Images coded for fluorescence intensity using a continuous grey scale (White highest, black lowest). 


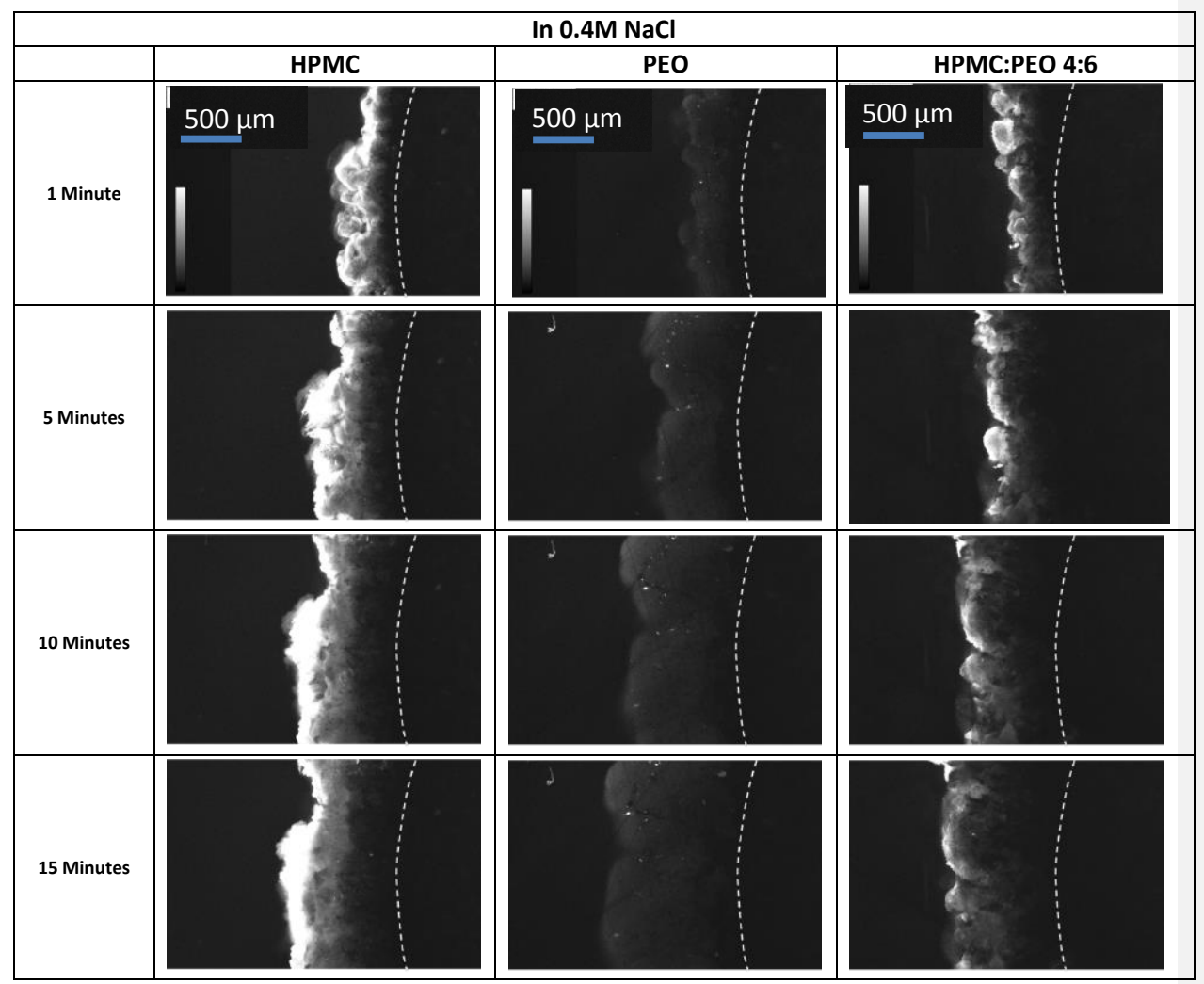

Figure 8: Early gel layer growth in $0.4 \mathrm{M} \mathrm{NaCl}$. Confocal fluorescence imaging at Ex $488 / \mathrm{Em} 510 \mathrm{~nm}$. Experiments conducted at $37 \pm 1{ }^{\circ} \mathrm{C}$ using $\mathrm{NaCl}$ media containing $0.008 \% \mathrm{w} / \mathrm{v}$ Congo red as a visualisation aid. Images coded for fluorescence intensity using a continuous grey scale (White highest, black lowest). 


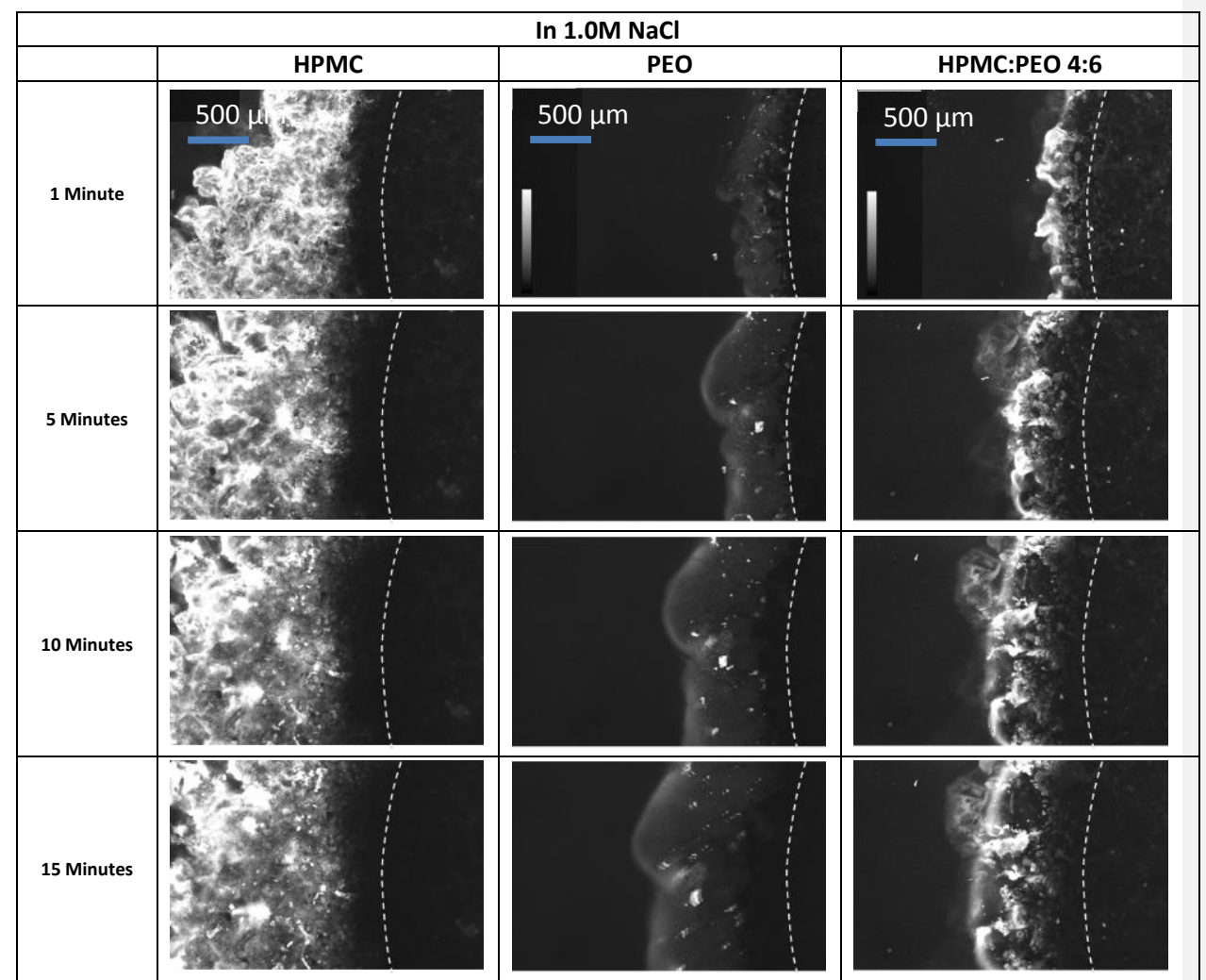

Figure 9: Early gel layer growth in $1.0 \mathrm{M} \mathrm{NaCl}$ Confocal fluorescence imaging at $\mathrm{Ex} 488 / \mathrm{Em} 510 \mathrm{~nm}$. Experiments conducted at $37 \pm 1{ }^{\circ} \mathrm{C}$ using $\mathrm{NaCl}$ media containing $0.008 \% \mathrm{w} / \mathrm{v}$ Congo red as a visualisation aid. Images coded for fluorescence intensity using a continuous grey scale (White highest, black lowest). 


\subsection{Internal matrix structure studied by digital optical imaging}

\subsubsection{Matrices hydrating in the absence of sodium chloride}

Figure 10 shows digital images of matrices hydrated up to 8 hours. Images were taken before and after sectioning to confirm that sectioning had not changed the dimensions of the hydrated matrix. Only in the case of two very weak gels were matrices distorted by sectioning and in these cases, matrix dimensions were measured from the unsectioned matrix, and sectioning was then used to confirm the presence or absence of a core. The images clearly show the swelling front that demarcates the hydrated gel layer from the 'dry' core, and we can also observe the overall shape of the matrix as hydration proceeds. Image analysis was used to quantify the thickness of the gel layer and visible core in the axial direction, form these images.

Figures 10 and 11 show the HPMC matrix undergoing hydration. It can be seen that: (i) the overall dimensions of the matrix grow over the whole 8 hour period; (ii) gel and core dimensions change little up to 4 hours; (iii) the gel layer then grows substantially with considerable loss of core between 6 and 8 hours; (iv) after 8 hours, the majority of matrix is gel, but which has sufficient gel strength to maintain a matrix shape. In contrast, the PEO matrix shows: (i) a more substantial loss of core after only 2 hours; (ii) swollen gel compensates for the loss of core and the matrix maintains its overall dimensions up to 4 hours, (iii) thereafter the matrix collapses, indicating that the gelled matrix after 6 hours is mechanically weak.

The 4:6 HPMC: PEO matrix appears to behave in a similar way to the HPMC matrix: (i) the gel and core dimensions are maintained up to 4 hours, (ii) there is reduced core at 6 hours but matrix dimensions are retained, (iii) after 8 hours the core has disappeared and the matrix shows a reduction in size.

These experiments illustrate how the addition of HPMC can change the hydration behaviour of a PEO matrix, to provide a longer retention of the core, and an increased gel strength which better maintains the matrix shape at later time points.

Whilst recognising that conditions are different with the dissolution test, these results suggest an explanation for the extended release properties and rank order (HPMC > 4:6 >PEO matrix) observed at later times in the dissolution tests. At earlier times, all 
matrices are similar in size and shape, and they possess a significant core. The similarity in surface area may explain their similar drug release kinetics during the early stages of dissolution. At later hydration times, however, dimensional changes were related to polymer composition. PEO matrices significantly erode, but substitution of HPMC with PEO in the 4:6 HPMC:PEO matrix show an increased thickness of the gel layer at 6 hours, which is again consistent with the observations from the MRI images shown in figure 5 . This would result in more prolonged diffusional release. This pattern may explain the drug release profiles in figure 4 and the longer $\mathrm{k}$ values in table 4.

The retention of an internal core is a critical feature in maintaining extended drug release in hydrophilic matrices [1]. In HPMC-containing matrices, the imaging results suggest acceleration in core hydration at 6 hours. We speculated that this may arise from internal weakening of the core allowing faster penetration of water. In PEO matrices, this effect was observed after only 2 hours. 


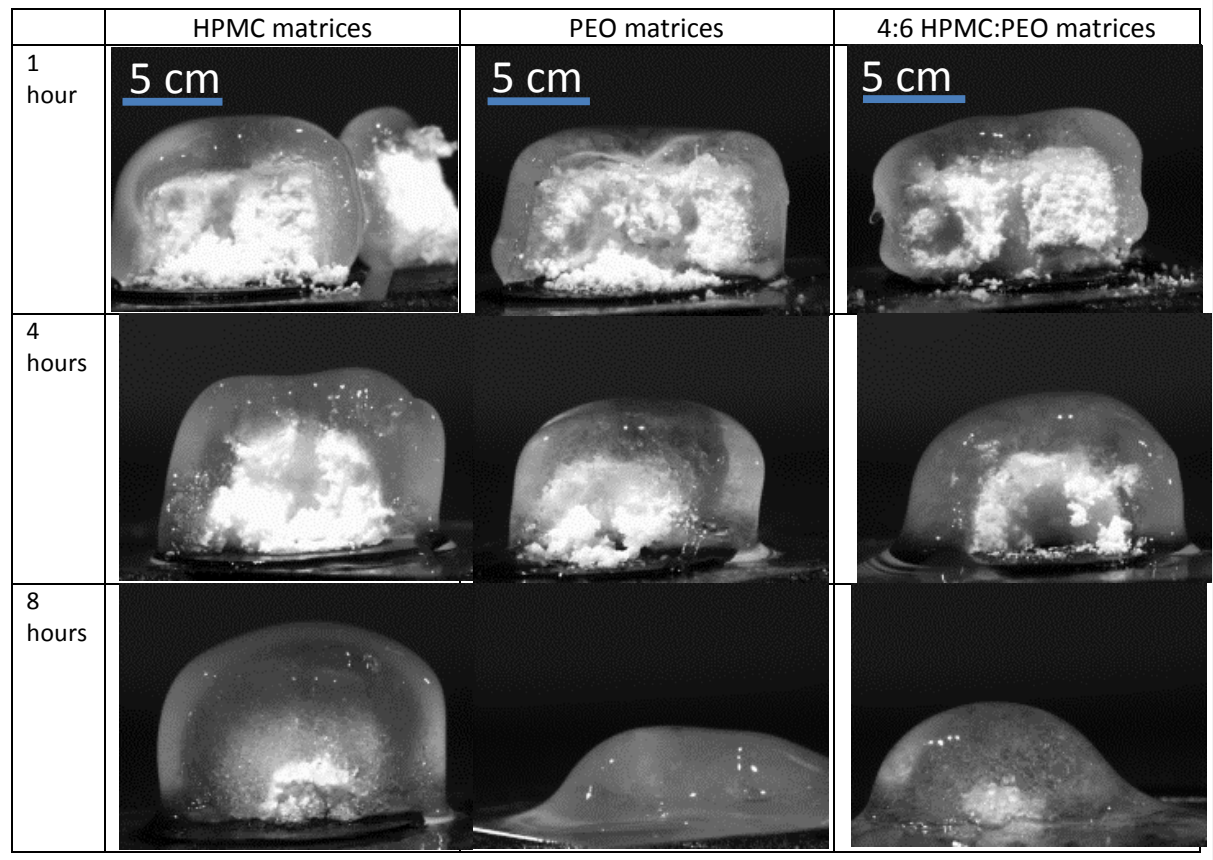

Figure 10: Digital images of sectioned HPMC PEO and 4:6 HPMC:PEO matrices showing the hydration behaviour over 8 hours in the absence of $\mathrm{NaCl}$. Experiments conducted at $37 \pm 1^{\circ} \mathrm{C}$, in pH 1.2 media. 

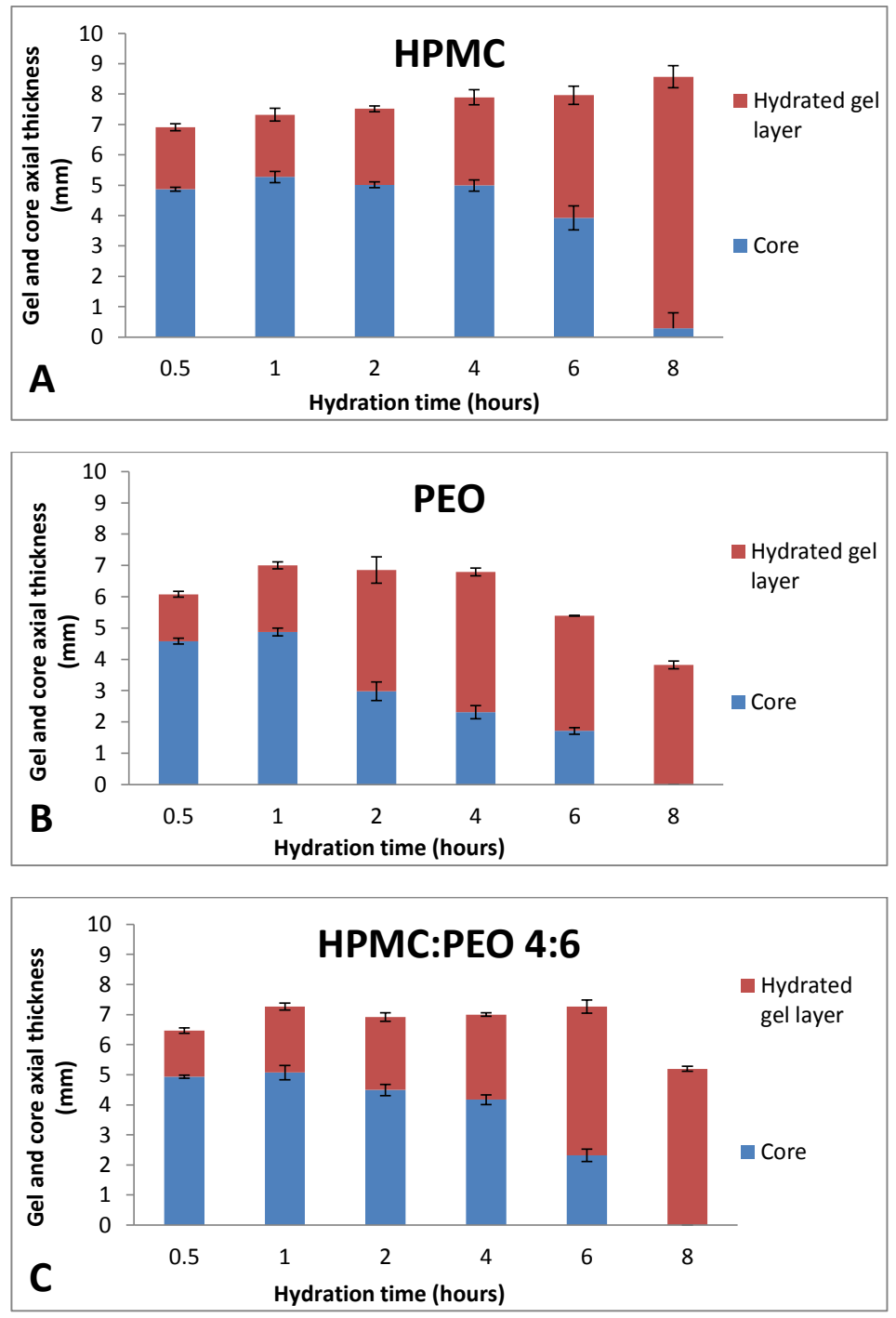

Figure 11: Changes in gel and core dimensions in matrices hydrating in the absence of $\mathrm{NaCl}$. Data obtained from image analysis of digital images of sectioned matrices hydrated at $37 \pm 1{ }^{\circ} \mathrm{C}$ in $\mathrm{pH} 1.2$ media. 


\subsubsection{Matrices hydrating in sodium chloride solutions}

Figures 12 and 13 show sectioned matrices and gel/core thickness data for matrices hydrating in $0.4 \mathrm{M} \mathrm{NaCl}$. The hydration pattern was very similar to matrices hydrating in the absence of $\mathrm{NaCl}$. Figures 14 and 15 show the same data for matrices hydrated in $1.0 \mathrm{M} \mathrm{NaCl}$. Under these extreme salt concentrations, the HPMC matrix had disintegrated before the one hour time point, but the PEO and 4:6 HPMC:PEO matrices appeared to be unaffected. The PEO matrix exhibits an enhanced gel thickness at 6 hours (figure 15), but still shows a significant collapse at 8 hours. The combined polymer matrix behaves like the PEO matrix in resisting the effects of extreme salt concentration, but there was no final collapse, and it maintained the overall dimensions of the matrix throughout the test. This provides evidence for enhanced gel strength, with HPMC clearly adding in some way to the robustness of the gel layer. 


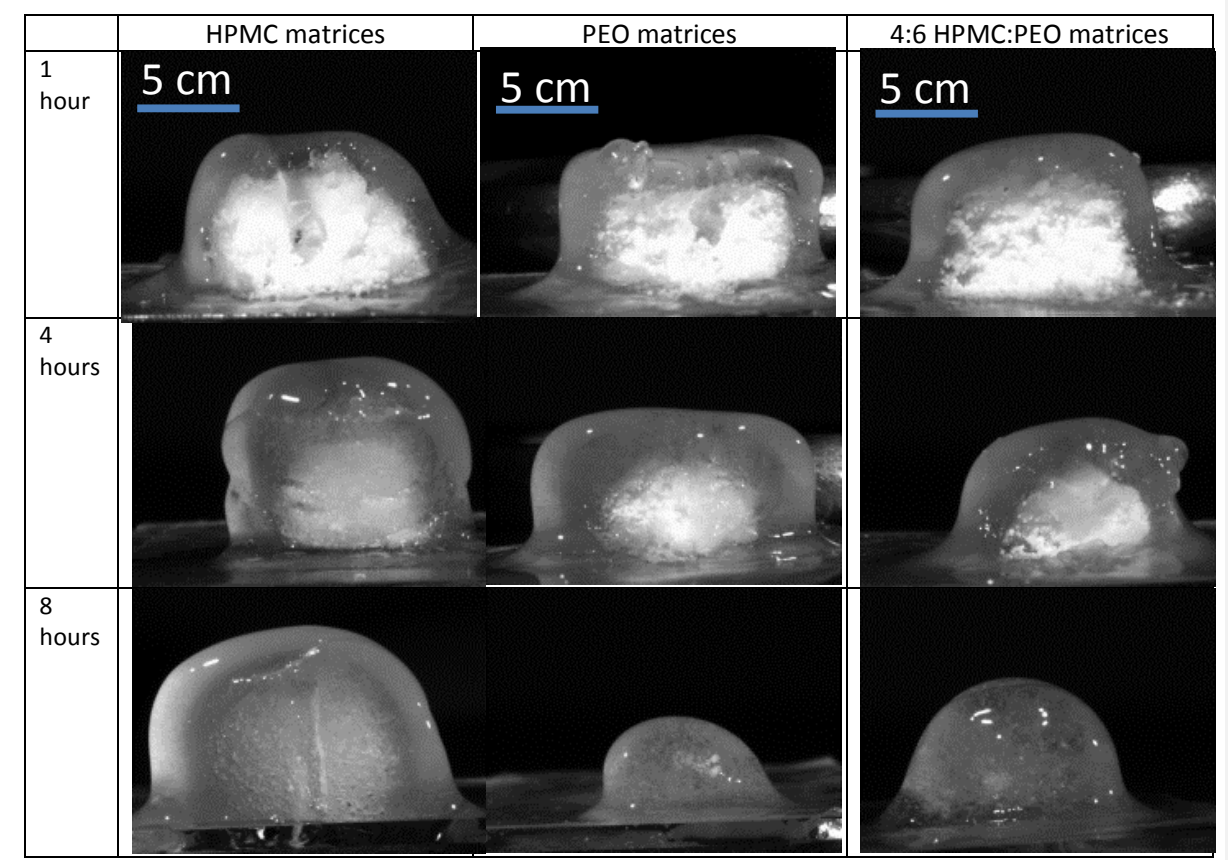

Figure 12: Digital images of sectioned HPMC PEO and 4:6 HPMC:PEO matrices showing the hydration behaviour over 8 hours in $0.4 \mathrm{M} \mathrm{NaCl}$. Experiments conducted at $37 \pm 1^{\circ} \mathrm{C}$, in pH 1.2 media. 

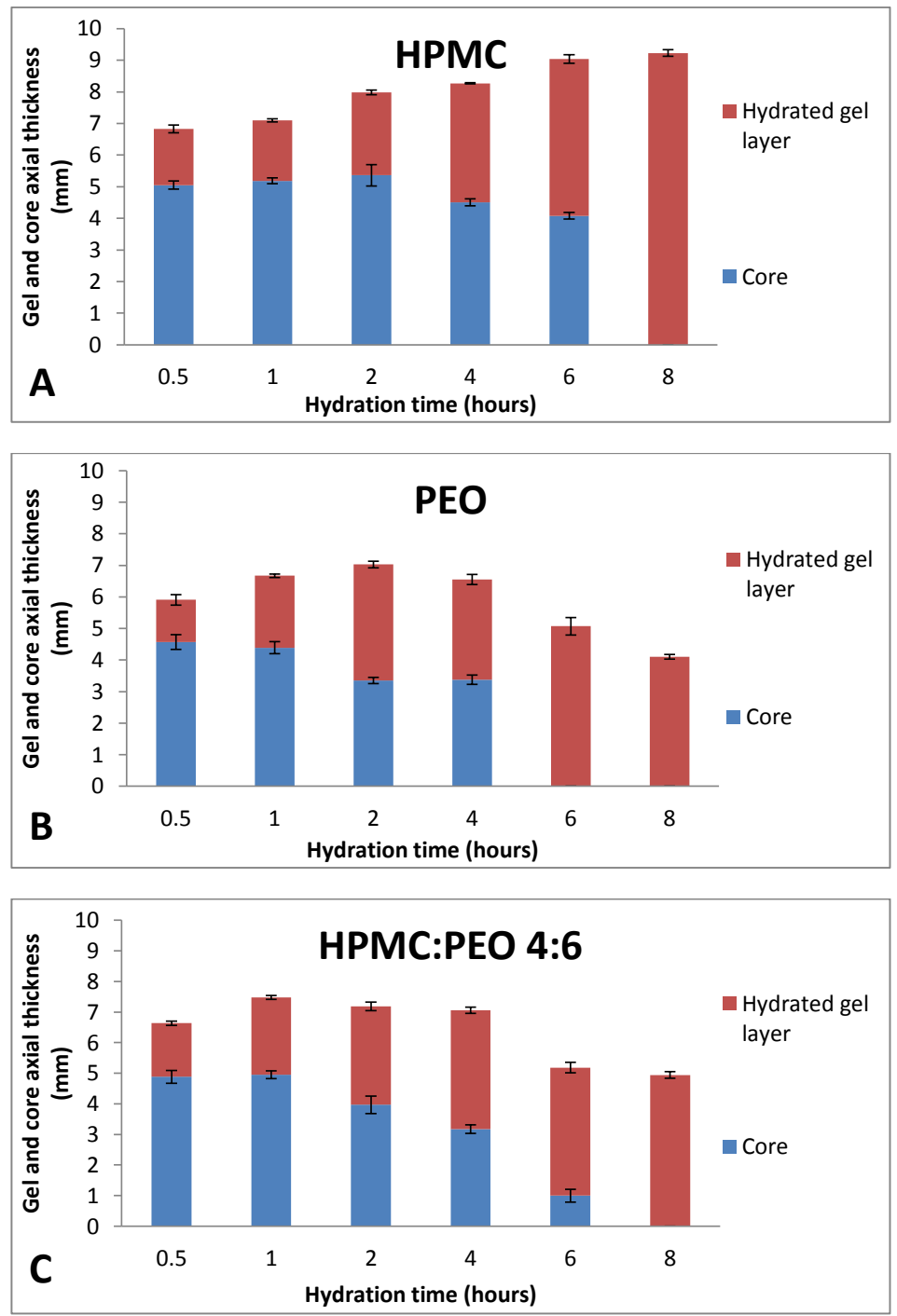

Figure 13: Changes in gel and core dimensions in matrices hydrating in $0.4 \mathrm{M} \mathrm{NaCl}$. Data obtained from image analysis of digital images of sectioned matrices hydrated at $37 \pm 1^{\circ} \mathrm{C}$ in $\mathrm{pH} 1.2$ media. 


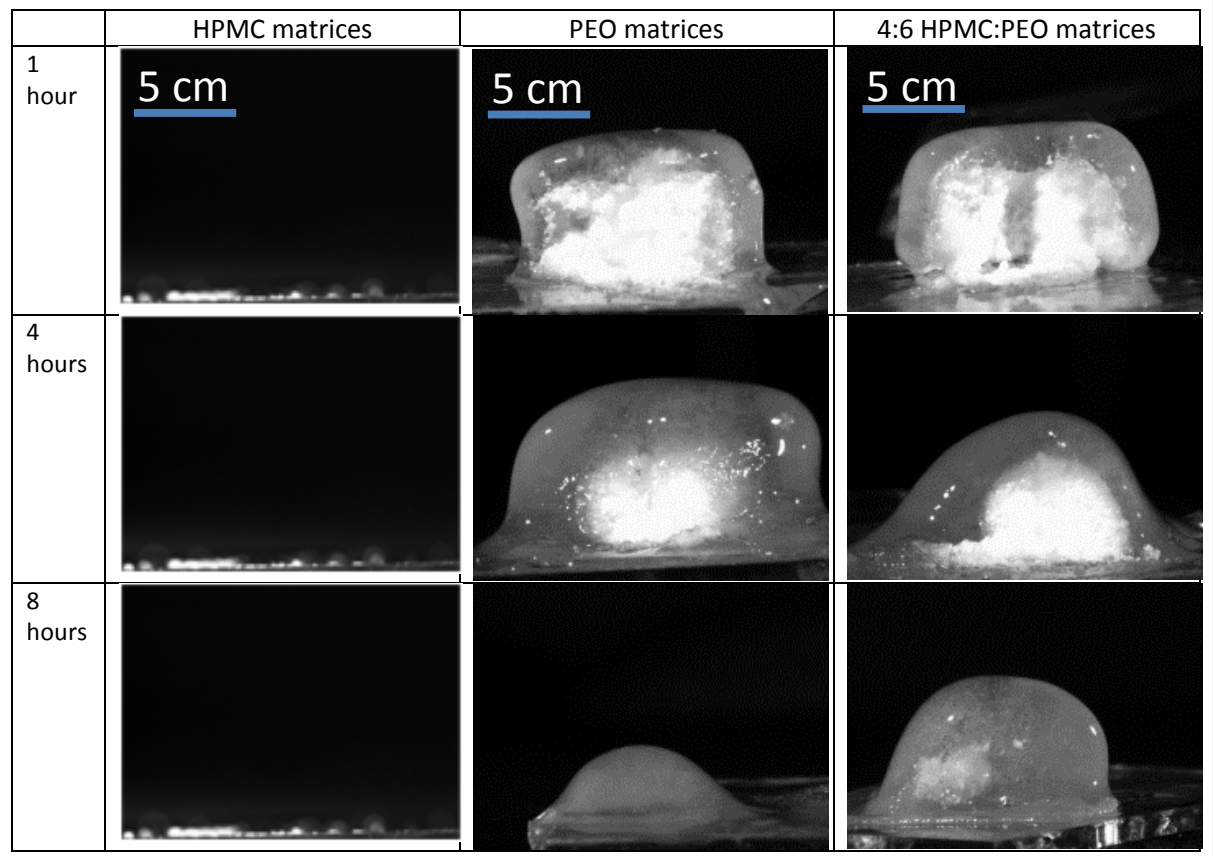

Figure 14: Digital images of sectioned HPMC PEO and 4:6 HPMC:PEO matrices showing the hydration behaviour over 8 hours in $1.0 \mathrm{M} \mathrm{NaCl}$. Experiments conducted at $37 \pm 1^{\circ} \mathrm{C}$, in $\mathrm{pH} 1.2$ media. 

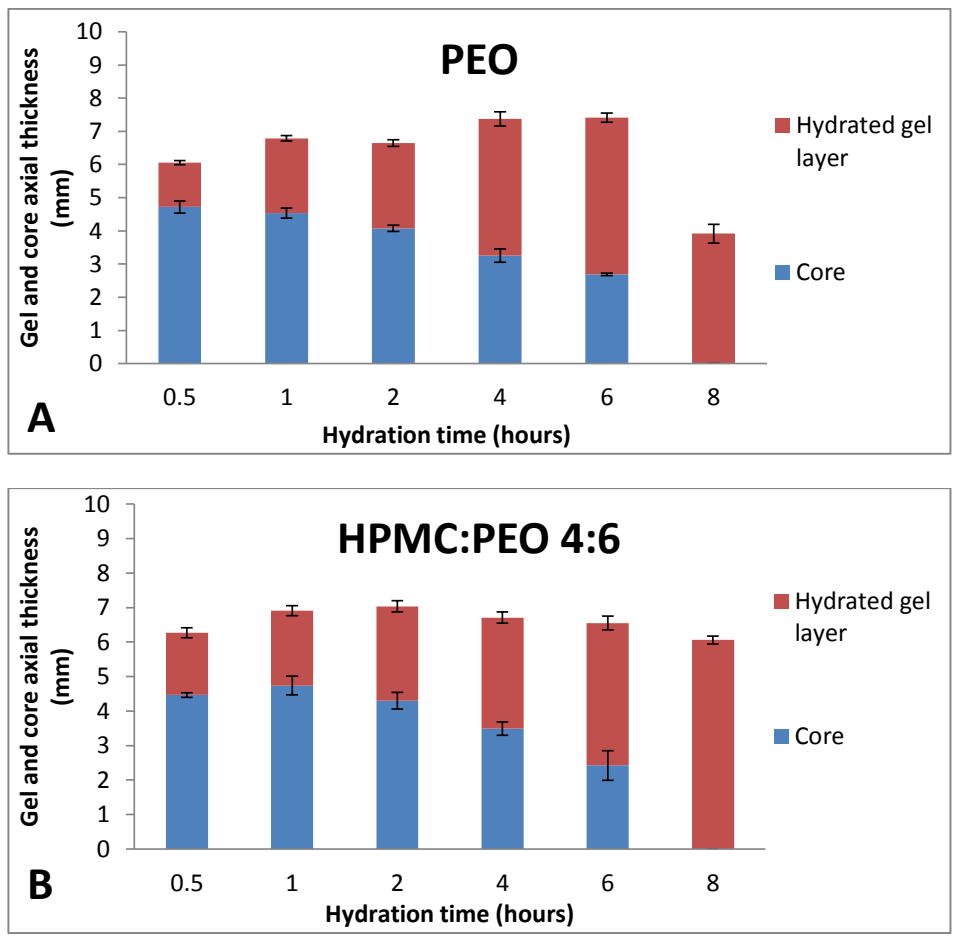

Figure 15: Changes in gel and core dimensions in matrices hydrating in 1.0M NaCl. Data obtained from image analysis of digital images of sectioned matrices hydrated at $37 \pm 1^{\circ} \mathrm{C}$ in $\mathrm{pH} 1.2$ media. 


\section{Conclusions}

Hydroxypropyl methylcellulose (HPMC) and polyethylene oxide (PEO) combinations can be used in hydrophilic matrices, to produce extended release dosage forms that combine the advantages of both polymers. The HPMC content of mixed polymer matrices provided longer extended release, reduced water penetration and matrix erosion, and increased the gel strength (seen particularly in the later stages of drug release) in comparison with a matrix containing PEO alone. The PEO content of the mixed polymer matrix reduced the matrix ionic strength sensitivity, in comparison to a matrix containing high proportions of HPMC. Mixed polymer matrices formed good gel layer diffusion barriers at all polymer ratios. In very high ionic strength media $(1.0 \mathrm{M} \mathrm{NaCl})$ where an HPMC matrix would disintegrate, the HPMC within the mixed polymer gel layer appeared to contribute to the extended release properties of the gel layer, possibly through adding tortuosity to the diffusional path of drug through the gel layer. MRI experiments correlate well with the optical microscopy data and their interpretation and provide additional information for different tablet formulations; MRI highlights the differences in hydration dynamics and subsequent gel layer formation which, in turn, allowed the behaviour of the UV-vis. drug release curves from USP 1 and USP 4 dissolution tests to be understood.

This work suggests that in future applications, mixed HPMC:PEO matrices may have a greater extended release robustness, in stomach environments containing foods with a high salt content mixed with other salts or soluble species that supress HPMC hydration. This would be especially so in circumstances where the matrices themselves have been formulated with high levels of ionic buffers, counter-ions or salts. 
1. P. Timmins, S.R. Pygall, C.D. Melia, Hydrophilic Matrix Tablets for Oral Controlled Release. AAPS Advances in Pharmaceutical Sciences Series, New York: Springer, 2014, p. 17-51.

2. R.S Harland, A. Garzzaniga, M.E. Sangalli, P. Colombo, N.A. Peppas, Drug Polymer Matrix Swelling and Dissolution. Pharmaceutical Research, 1988. 5(8): p. 488-494.

3. P. Colombo, R. Bettini, G. Massimo,PL. Catellani, P. Santi, NA. Peppas, Drug Diffusion Front Movement Is Important in Drug-Release Control from Swellable Matrix Tablets. J Pharm Sci, 1995. 84(8): p. 991-997.

4. P. Colombo, R. Bettini, P. Santi, N.A, Peppas, Swellable matrices for controlled drug delivery: gel-layer behavior, mechanisms and optimal performance. Pharm. Sci. Technol. Today, 2000. 3: p. 198-204.

5. A. Korner, A. Larsson, A. Andersson, L. Piculell, Swelling and polymer erosion for poly(ethylene oxide) tablets of different molecular weights polydispersities. J Pharm Sci, 2010. 99(3): p. 1225-38.

6. S.A. Modi, P.D.Gaikwad, V. H. Bankar, S. P. Pawar, Sustained release drug delivery system : a review. International Journal of Pharma. Research \& Develpment - Online, 2011. 2.

7. J.T. Leskinen, MA. Hakulinen, M. Kuosmanen, J. Ketolainen, S. Abrahmsén-Alami, R Lappalainen. Monitoring of swelling of hydrophilic polymer matrix tablets by ultrasound techniques. International Journal of Pharmaceutics, 2011. 404(1-2): p. 142-147.

8. K. Mitchell, J.L. Ford, D.J. Armstrong, P.N.C. Elliott, C. Rostron, J. E. Hogan, The Influence of Additives on the Cloud Point, Disintegration and Dissolution of Hydroxypropylmethylcellulose Gels and Matrix Tablets. International Journal of Pharmaceutics, 1990. 66(1-3): p. 233-242.

9. H.D. Williams, R. Ward, I.J. Hardy, C.D. Melia, The effect of sucrose and salts in combination on the drug release behaviour of an HPMC matrix. European Journal of Pharmaceutics and Biopharmaceutics, 2010. 76(3): p. 433-6.

10. H.D. Williams, R. Ward, I.J. Hardy, C.D. Melia, The extended release properties of HPMC matrices in the presence of dietary sugars. Journal of Control Release, 2009. 138(3): p. 251-9.

11. H.D.Williams, R. Ward, I.J. Hardy, C.D. Melia, The effect of sucrose and salts in combination on the drug release behaviour of an HPMC matrix. European Journal of Pharmaceutics and Biopharmaceutics, 2010. 76(3): p. 433-6.

12. L. Herbert, N.G. Lordi, Some factors affecting the release of a water-soluble drug from a compressed hydrophilic matrix. Journal of Pharmaceutical Sciences, 1966. 55(8): p. 840-843.

13. G.S. Bajwa, K. Hoebler, C. Sammon, P. Timmins, C.D. Melia, Microstructural imaging of early gel layer formation in HPMC matrices. Journal of Pharmaceutical Sciences, 2006. 95(10): p. 2145-57.

14. A.R. RajabiSiahboomi, R. Bowtell, P. Mansfield, C.D. Melia, Structure and behavior in hydrophilic matrix sustained release dosage forms .4. Studies of water mobility and diffusion coefficients in the gel layer of HPMC tablets using NMR imaging. Pharmaceutical Research, 1996. 13(3): p. 376-380.

15. A.E. Royce, Directly compressible polyethylene oxide vehicle for preparing therapeutic dosage forms. 1993: USA.

16. C.J. Kim, Drug release from compressed hydrophilic Polyox ${ }^{\circledR}$ WSR tablets. Journal of Pharmaceutical Sciences, 1995. 84(3): p. 303-306.

17. C.J.Kim, Effects of drug solubility, drug loading, and polymer molecular weight on drug release from polyox (R) tablets. Drug Development and Industrial Pharmacy, 1998. 24(7): $p$. 645-651.

18. L. Maggi, R. Bruni, and U. Conte, High molecular weight polyethylene oxides (PEOs) as an alternative to HPMC in controlled release dosage forms. International Journal of Pharmaceutics, 2000. 195(1-2): p. 229-238.

19. S. Turner, M. Hite, R. Fassihi, Formulation development and human in vitro-in vivo correlation for a novel, monolithic controlled-release matrix system of high load and highly 
water-soluble drug niacin. Drug Development and Industrial Pharmacy, 2004. 30(8): p. 797807.

20. P.S. Wray, G.S. Clarke, S.G. Kazarian, Dissolution of tablet-in-tablet formulations studied with ATR-FTIR spectroscopic imaging. European Journal of Pharmaceutical Sciences, 2013. 48(4-5): p. 748-757.

21. A. Jayan, Investigating the drug release mechanisms of mixed HPMC/PEO hydrophilic matrices, in University of Nottingham 2001, University of Nottingham University of Nottingham

22. G. Gusler, Mei Chau, Padua, Optimal polymer mixtures for gastric retentive tablets, U.S. Patent, 2004: USA.

23. P.L. Ritger, N.A. Peppas., A simple equation for description of solute release II. Fickian and anomalous release from swellable devices. Journal of Controlled Release 1987. 5: p. 37-42.

24. J. Siepmann, N.A. Peppas, Modeling of drug release from delivery systems based on hydroxypropyl methylcellulose (HPMC). Adv Drug Deliv Rev, 2001. 48(2-3): p. 139-57.

25. E.B. Wong, Partially Pregelatinized Starch as an Excipient in HPMC Matrices, in School of Pharmacy 2009, University of Nottingham University of Nottingham

26. A. Viriden, A. Larsson, H. Schagerlöf, B. Wittgren, Model drug release from matrix tablets composed of HPMC with different substituent heterogeneity. International Journal of Pharmaceutics, 2010. 401(1-2): p. 60-7.

27. YY. Chen, LP. Hughes, LF Gladden, MD, Mantle, Quantitative ultra-fast MRI of HPMC swelling and dissolution. Journal of Pharmaceutical Sciences, 2010. 99(8): p. 3462-72.

28. Q. Zhang, L. Gladden, P. Avalle, M. Mantle, In vitro quantitative $((1)) \mathrm{H}$ and $((19)) \mathrm{F}$ nuclear magnetic resonance spectroscopy and imaging studies of fluvastatin in Lescol(R) XL tablets in a USP-IV dissolution cell. Journal of Control Release, 2011. 156(3): p. 345-54.

29. G. Shiko, LF. Gladden, AJ. Sederman, PC. Connolly, JM. Butler, MRI studies of the hydrodynamics in a USP 4 dissolution testing cell. Journal of Pharmaceutical Sciences, 2011. 100(3): p. 976-91.

30. P. Colombo, R. Bettini, P. Santi, A. De Ascentiis, N.A. Peppas, Analysis of the swelling and release mechanisms from drug delivery systems with emphasis on drug solubility and water transport. Journal of Controlled Release, 1996. 39(2-3): p. 231-237.

31. T. Hino, J.L. Ford, Effect of nicotinamide on the properties of aqueous HPMC solutions. International Journal of Pharmaceutics, 2001. 226(1-2): p. 53-60.

32. A.R. Rajabi-Siahboomi, hydroxypropylmethylcellulose in hydrophilic matrix dosage forms, in School of Pharmacy. 1993, University of Nottingham: Nottingham.

33. C.B. McCrystal, J.L. Ford, A.R. Rajabi-Siahboomi, Water distribution studies within cellulose ethers using differential scanning calorimetry. 1 . Effect of polymer molecular weight and drug addition. Journal of Pharmaceutical Sciences 1999. 88(8): p. 792-796.

34. C.B. McCrystal, J.L. Ford, A.R. Rajabi-Siahboomi, Water distribution studies within cellulose ethers using differential scanning calorimetry. 2. Effect of polymer substitution type and drug addition. Journal of Pharmaceutical Sciences, 1999. 88(8): p. 797-801.

35. S.Q. Liu, S.C. Joshi, Y.C. Lam, Effects of salts in the Hofmeister series and solvent isotopes on the gelation mechanisms for hydroxypropylmethylcellulose hydrogels. Journal of Applied Polymer Science, 2008. 109(1): p. 363-372.

36. S.C. Joshi, Sol-Gel Behavior of Hydroxypropyl Methylcellulose (HPMC) in lonic Media Including Drug Release. Materials, 2011. 4(10): p. 1861-1905.

37. E. Florin, R. Kjellander, J.C. Eriksson, Salt Effects on the Cloud Point of the Poly(Ethylene Oxide) + Water-System. Journal of the Chemical Society-Faraday Transactions I, 1984. 80: $p$. 2889-2910

38. A.R. Rajabi-Siahboomi, M.Levina, T.P. Farrell, D. Palmer, The Influence of Hydro-Alcoholic Media on Drug Release. Pharmaceutical Technology, 2011. 35(7). 
39. J. Lopes, J. Pinto, P. Costa, Compressed matrix core tablet as a quick/slow dual-component delivery system containing ibuprofen. Aaps Pharmscitech, 2007. 8(3).

40. C.D. Melia, A.R. Rajabi-Siahboomi, R.W. Bowtell, Magnetic resonance imaging of controlled release pharmaceutical dosage forms. Pharmaceutical Science \& Technology Today, 1998. 1(1): p. 32-39.

41. S. Missaghi, P. Patel, TP. Farrell, H. Huatan, AR. Rajabi-Siahboomi. Investigation of critical core formulation and process parameters for osmotic pump oral drug delivery. Aaps Pharmscitech, 2014. 15(1): p. 149-60.

42. M.L. Mirza, J. Iqbal, H. Aziz, Sorption of congo red on cellulose. Journal of the Chemical Society of Pakistan, 1996. 18(3): p. 233-236.

43. H.D. Williams, R. Ward, A.Culy, I.J. Hardy, C.D. Melia, Designing HPMC matrices with improved resistance to dissolved sugar. International Journal of Pharmaceutics, 2010. 401(12): p. 51-59. 